MATHEMATICS OF COMPUTATION

Volume 76, Number 259, July 2007, Pages 1195-1217

S 0025-5718(07)01929-1

Article electronically published on March 15, 2007

\title{
STABILIZED FINITE ELEMENT METHOD FOR NAVIER-STOKES EQUATIONS WITH PHYSICAL BOUNDARY CONDITIONS
}

\author{
M. AMARA, D. CAPATINA-PAPAGHIUC, AND D. TRUJILLO
}

\begin{abstract}
This paper deals with the numerical approximation of the 2D and 3D Navier-Stokes equations, satisfying nonstandard boundary conditions. This lays on the finite element discretisation of the corresponding Stokes problem, which is achieved through a three-fields stabilized mixed formulation. A priori and a posteriori error bounds are established for the nonlinear problem, ascertaining the convergence of the method. Finally, numerical tests are presented, including mesh refinement via error indicators.
\end{abstract}

\section{INTRODUCTION}

We are interested in this paper in the stationary Navier-Stokes problem satisfying physical boundary conditions (see also [10]) in a simply connected bounded domain $\Omega$ of $\mathbb{R}^{2}$, with a polygonal boundary $\Gamma=\partial \Omega$.

The corresponding 2D Stokes equations with the same boundary conditions were studied in 12 and 4, by means of different three-fields variational formulations. In [4, after showing that the new vorticity-velocity-pressure formulation proposed was well-posed, the authors discretized it by means of conforming low-order finite elements. The discrete inf-sup condition is then obvious, while the discrete coercivity is obtained by adding a stabilization term (see [1) taking into account the jumps of both the vorticity and the pressure across the edges of the triangulation. Error bounds were deduced in a technical way, ensuring the unconditional convergence of the method as well as an optimal convergence rate $O(h)$ whenever the exact solution is sufficiently smooth.

The goal of the present work is to propose a well-posed and convergent numerical approximation, as well as a priori and a posteriori error estimates, for the NavierStokes equations. The discretization is based on the method developed in [4] for the associated Stokes equations, the present paper being a generalization to the nonlinear case. However, we consider here a simpler discrete formulation of the Stokes equations, in which we stabilize only the pressure and which yields similar results from both theoretical and numerical points of view.

To deal with the nonlinear aspects of the problem, we use a variant of the implicit function theorem which can be found for instance in [15. We thus obtain existence and uniqueness of the solution of the discrete problem in a neighbourhood of the exact solution, unconditional convergence of the approximation of the Navier-Stokes

Received by the editor June 4, 2004 and, in revised form, July 6, 2005.

2000 Mathematics Subject Classification. Primary 35Q30, 65N12; Secondary 65N30.

(C)2007 American Mathematical Society Reverts to public domain 28 years from publication 
equations and also a priori and a posteriori error estimates for the vorticity and the pressure in $L^{2}(\Omega)$, respectively for the velocity in $\mathbf{L}^{4}(\Omega)$. For smooth solutions, one gets the same convergence rate $O(h)$ as for the Stokes problem.

Here we develop the bidimensional case; the extension to $3 \mathrm{D}$ together with $3 \mathrm{D}$ numerical examples are presented in [3. Several numerical experiments are shown in the last section. On the one hand an academic test is considered, highlighting the theoretical results on the convergence rates for different Reynolds numbers. On the other hand, more realistic cases are treated by employing a posteriori error estimators.

The paper is organized as follows. In section 2 we introduce the mathematical framework for the Stokes and Navier-Stokes problems with nonstandard boundary conditions, and in section 3 we study the discrete Stokes operator. In particular, we prove that it satisfies a stability condition as well as a consistency condition. Thanks to these properties, we establish in section 4 that the discrete Navier-Stokes problem is well-posed and we obtain error bounds for the solution which allow us to deduce the unconditional convergence of the approximation and its convergence rate. We also propose an a posteriori error indicator. Finally, the last section deals with the numerical experiments.

\section{FUnCTIONAL FRAMEWORK}

2.1. The model problem. In order to describe our model problem, let us first introduce some notations. For any $2 D$ vector field $\mathbf{v}=\left(v_{1}, v_{2}\right)^{t}$, we denote

$$
\mathbf{v}^{\perp}=\left(-v_{2}, v_{1}\right)^{t}, \quad \operatorname{div} \mathbf{v}=\partial_{1} v_{1}+\partial_{2} v_{2}, \quad \text { curl } \mathbf{v}=\partial_{1} v_{2}-\partial_{2} v_{1}
$$

and, for any scalar field $\phi, \operatorname{curl} \phi=\left(\partial_{2} \phi,-\partial_{1} \phi\right)^{t}$. We suppose that $\Gamma$ is composed of three open and disjoint subsets $\Gamma_{1}, \Gamma_{2}, \Gamma_{3}$ such that $\Gamma=\bar{\Gamma}_{1} \cup \bar{\Gamma}_{2} \cup \bar{\Gamma}_{3}$ and, for the sake of simplicity, we suppose that $\left|\Gamma_{2}\right|>0$ where $|\cdot|$ denotes the Lebesgue measure. We denote, as usually, by $\mathbf{n}$ the unit outward normal vector to the boundary $\Gamma$ and by $\mathbf{t}$ the associated unit tangent vector. We agree to denote the vector spaces by bold letters; in particular for real numbers $p>1,0<s \leq 1$ we denote $L^{p}(\Omega)^{2}, H^{s}(\Omega)^{2}$ by $\mathbf{L}^{p}(\Omega), \mathbf{H}^{s}(\Omega)$ respectively. The notation $\|\cdot\|_{\mathcal{L}(Y)}$ stands for the norm of a linear continuous operator of $\mathcal{L}(Y)$, where $Y$ is a Banach space.

We consider the stationary incompressible Navier-Stokes equations

$$
\left\{\begin{array}{cc}
\mathbf{u} \nabla \mathbf{u}-\nu \Delta \mathbf{u}+\nabla p=\mathbf{f} & \text { in } \Omega, \\
\operatorname{div} \mathbf{u}=0 & \text { in } \Omega
\end{array}\right.
$$

and impose the following boundary conditions which are described, for instance, in [10],

$$
\left\{\begin{array}{lll}
\mathbf{u} \cdot \mathbf{n}=0, & \mathbf{u} \cdot \mathbf{t}=0 & \text { on } \Gamma_{1} \\
\mathbf{u} \cdot \mathbf{t}=0, & p+\frac{1}{2} \mathbf{u} \cdot \mathbf{u}=0 & \text { on } \Gamma_{2} \\
\mathbf{u} \cdot \mathbf{n}=0, & \omega=0 & \text { on } \Gamma_{3}
\end{array}\right.
$$

where $\omega=$ curlu represents the scalar vorticity.

Remark 2.1. In order to simplify the presentation and without losing generality, here we consider homogeneous boundary conditions for the pressure and the vorticity. The nonhomogeneous case is treated in [2. However, in the last section, numerical tests are also carried out for nonhomogeneous boundary conditions. 
By means of the dynamic pressure $\widetilde{p}=p+\frac{1}{2} \mathbf{u} \cdot \mathbf{u}$ and of the relation

$$
\mathbf{u} \nabla \mathbf{u}+\nabla p=\omega \mathbf{u}^{\perp}+\nabla \tilde{p},
$$

the previous problem can be written as

$$
\begin{cases}\nu \operatorname{curl} \omega+\nabla \widetilde{p}+\omega \mathbf{u}^{\perp}=\mathbf{f} & \text { in } \Omega, \\ \omega=\text { curl } \mathbf{u} & \text { in } \Omega, \\ \operatorname{div} \mathbf{u}=0 & \text { in } \Omega,\end{cases}
$$

together with the boundary conditions

$$
\left\{\begin{array}{lll}
\mathbf{u} \cdot \mathbf{n}=0, & \mathbf{u} \cdot \mathbf{t}=0 & \text { on } \Gamma_{1} \\
\mathbf{u} \cdot \mathbf{t}=0, & \widetilde{p}=0 & \text { on } \Gamma_{2} \\
\mathbf{u} \cdot \mathbf{n}=0, & \omega=0 & \text { on } \Gamma_{3}
\end{array}\right.
$$

The unknowns are now the velocity field $\mathbf{u}$ and the scalar fields $\omega$ and $\tilde{p}$ (denoted by $p$ in the following). The kinematic viscosity $\nu>0$ is given, and for the sake of simplicity, the force field $\mathbf{f}$ is taken in $\mathbf{L}^{4 / 3}(\Omega)$.

2.2. The linear Stokes problem. The analysis of the nonlinear Navier-Stokes problem uses the properties of the associated linear Stokes operator. Therefore, we consider the Stokes equations

$$
\begin{cases}\nu \operatorname{curl} \omega+\nabla p=\mathbf{g} & \text { in } \Omega, \\ \omega=\operatorname{curl} \mathbf{u} & \text { in } \Omega, \\ \operatorname{div} \mathbf{u}=0 & \text { in } \Omega,\end{cases}
$$

endowed with the same type of boundary conditions as in (2.2),

$$
\left\{\begin{array}{lll}
\mathbf{u} \cdot \mathbf{n}=0, & \mathbf{u} \cdot \mathbf{t}=0 & \text { on } \Gamma_{1} \\
\mathbf{u} \cdot \mathbf{t}=0, & p=0 & \text { on } \Gamma_{2} \\
\mathbf{u} \cdot \mathbf{n}=0, & \omega=0 & \text { on } \Gamma_{3}
\end{array}\right.
$$

where $\mathbf{g} \in \mathbf{L}^{4 / 3}(\Omega)$.

A three-fields variational formulation was introduced in 4, for smoother forces $\mathbf{g} \in \mathbf{L}^{2}(\Omega)$. Let us next recall some results established in $[4$ and adapt them to the case where $\mathbf{g} \in \mathbf{L}^{4 / 3}(\Omega)$.

One first needs to define the following Hilbert spaces:

$$
\begin{aligned}
\mathbf{M} & =\left\{\mathbf{v} \in \mathbf{H}(\text { div }, \text { curl } ; \Omega) ;\left.\quad \mathbf{v} \cdot \mathbf{n}\right|_{\Gamma_{1} \cup \Gamma_{3}}=\left.\mathbf{v} \cdot \mathbf{t}\right|_{\Gamma_{1} \cup \Gamma_{2}}=0\right\} \\
\mathbf{X} & =\mathbf{L}^{2}(\Omega)
\end{aligned}
$$

where

$$
\mathbf{H}(\operatorname{div}, \text { curl } ; \Omega)=\left\{\mathbf{v} \in \mathbf{L}^{2}(\Omega) ; \operatorname{div} \mathbf{v} \in L^{2}(\Omega), \operatorname{curl} \mathbf{v} \in L^{2}(\Omega)\right\} .
$$

The spaces $\mathbf{H}($ div, curl; $\Omega), \mathbf{M}$ are both normed by $\|\mathbf{v}\|_{\mathbf{M}}=\left(\|\mathbf{v}\|_{0, \Omega}^{2}+\|\right.$ div $\mathbf{v} \|_{0, \Omega}^{2}+$ $\left.\|\operatorname{curl} \mathbf{v}\|_{0, \Omega}^{2}\right)^{1 / 2}$.

We assume in this paper that

$$
\{\mathbf{v} \in \mathbf{M} ; \operatorname{div} \mathbf{v}=\operatorname{curl} \mathbf{v}=0 \quad \text { a.e. in } \Omega\}=\{\mathbf{0}\} .
$$

The hypothesis (H1) is true in particular if one of the following situations hold: $\left|\Gamma_{1}\right|>0$, or $\left|\Gamma_{1}\right|=\left|\Gamma_{3}\right|=0$, or $\left|\Gamma_{1}\right|=0$ and $\left|\Gamma_{3}\right|>0$ with $\Gamma_{3}$ simply connected. Then one gets (according to [4]) that the seminorm $|\mathbf{v}|_{\mathbf{M}}=\left(\|\operatorname{div} \mathbf{v}\|_{0, \Omega}^{2}+\right.$ $\|$ curl $\left.\mathbf{v} \|_{0, \Omega}^{2}\right)^{1 / 2}$ is a norm on $\mathbf{M}$, equivalent to the norm $\|\cdot\|_{\mathbf{M}}$. 
Another key point is that in what follows, the space $\mathbf{M}$ is assumed to be continuously imbedded in $\mathbf{H}^{s}(\Omega)$, for some $\left.\left.s \in\right] 1 / 2,1\right]$. This is not a restrictive hypothesis; it is satisfied (see [11]) if there are no nonconvex corners (i.e., corners of $\Omega$ where the openings of the angles of $\Omega$ are larger than $\pi$ ) at the intersection of $\bar{\Gamma}_{1} \cup \bar{\Gamma}_{2}$ and $\bar{\Gamma}_{3}$.

The previous statement holds with $s=1$ if $\Omega$ is a convex polygon. Taking $\Omega$ as a Lipschitz-continuous domain and $\left|\Gamma_{2}\right|=\left|\Gamma_{3}\right|=0$ also yields the optimal case $s=1$ (cf. for instance [13]).

Then on the one hand, Sobolev's imbedding theorem implies that $\mathbf{M}$ is also continuously imbedded in $\mathbf{L}^{4}(\Omega)$ and on the other hand, we see that the traces of its elements belong to $\mathbf{L}^{2}(\Gamma)$. Thus, the linear form

$$
l(\mathbf{v})=-\int_{\Omega} \mathbf{g} \cdot \mathbf{v} d \Omega
$$

is well defined on $\mathbf{M}$, for any $\mathbf{g} \in \mathbf{L}^{4 / 3}(\Omega)$. Following 4 , we next define for all $\sigma=(\omega, p), \tau=(\theta, q) \in \mathbf{X}$ and $\mathbf{v} \in \mathbf{M}$, the bilinear forms

$$
\begin{aligned}
a(\sigma, \tau) & =\nu \int_{\Omega} \omega \theta d \Omega, \\
b(\tau, \mathbf{v}) & =-\nu \int_{\Omega} \theta \operatorname{curl} \mathbf{v} d \Omega+\int_{\Omega} q d i v \mathbf{v} d \Omega,
\end{aligned}
$$

and consider the mixed variational formulation of the Stokes problem:

$$
\left\{\begin{array}{cl}
\text { Find }(\sigma, \mathbf{u}) \in \mathbf{X} \times \mathbf{M}, & \\
a(\sigma, \tau)+b(\tau, \mathbf{u})=0 & \forall \tau \in \mathbf{X}, \\
b(\sigma, \mathbf{v})=l(\mathbf{v}) & \forall \mathbf{v} \in \mathbf{M} .
\end{array}\right.
$$

In [4, problem (2.3) was shown to satisfy the Babuška-Brezzi conditions (cf. [6] for instance), so (2.3) has a unique solution.

Remark 2.2. Let us note that one may also take $\mathbf{g} \in \mathbf{M}^{\prime}$ and still obtain the well-posedness of the Stokes problem.

Then one can define a linear continuous operator

$$
S: \mathbf{L}^{4 / 3}(\Omega) \rightarrow \mathbf{X} \times \mathbf{L}^{4}(\Omega), \quad S(\mathbf{g})=(\sigma, \mathbf{u})
$$

where $(\sigma, \mathbf{u})$ is the unique solution of the Stokes problem (2.3).

For the simplicity of notation, we shall denote from now on the Banach space $\mathbf{X} \times \mathbf{L}^{4}(\Omega)$ by $\mathbf{Y}$.

2.3. The nonlinear Navier-Stokes problem. By introducing the nonlinear operator

$$
G: \mathbf{Y} \rightarrow \mathbf{L}^{4 / 3}(\Omega), \quad G(\sigma, \mathbf{u})=\omega \mathbf{u}^{\perp},
$$

the Navier-Stokes equations (2.1) with homogeneous boundary conditions can be put in the general setting of a nonlinear problem as follows:

$$
F(\sigma, \mathbf{u})=(\mathbf{0}, \mathbf{0}) \text {. }
$$

The mapping $F$ is defined by

$$
F: \mathbf{Y} \rightarrow \mathbf{Y}, \quad F(\tau, \mathbf{v})=(\tau, \mathbf{v})-S(\mathbf{f}-G(\tau, \mathbf{v})) .
$$

We assume in what follows that there exists a solution $(\sigma, \mathbf{u})$ such that $F(\sigma, \mathbf{u})=$ 0 and $D F(\sigma, \mathbf{u})$ is an isomorphism of $\mathbf{X} \times \mathbf{L}^{4}(\Omega)$. It is well known that the classical 
Navier-Stokes problem admits at least a solution $(\sigma, \mathbf{u})$, the uniqueness holding under a hypothesis of small data; in this last case, $D F(\sigma, \mathbf{u})$ is an isomorphism.

\section{Finite element discretization of the Stokes Problem}

We are next interested in the numerical approximation of the nonlinear problem (2.4). For this purpose, one first needs to consider a discretization of the associated linear problem (2.3).

Let $\left(\mathcal{T}_{h}\right)_{h>0}$ be a regular family of triangulations of $\bar{\Omega}$ consisting of triangles. We classically denote by $h_{K}$ the diameter of the triangle $K$, by $h=\max _{K \in T_{h}} h_{K}$, by $\mathcal{E}_{h}$ the set of internal edges and by $h_{e}$ the length of the edge $e$. We assume that each triangulation is compatible with the boundary conditions and moreover, that $\Gamma_{2}$ contains at least one vertex.

For the approximation of the mixed variational formulation (2.3), we consider the same finite dimensional spaces as in [4, that is:

$$
\begin{aligned}
L_{h} & =\left\{q \in L^{2}(\Omega) ; q_{\mid K} \in P_{0}(K) \forall K \in \mathcal{T}_{h}\right\}, \\
\mathbf{X}_{h} & =L_{h} \times L_{h}, \\
\mathbf{M}_{h} & =\left\{\mathbf{v} \in\left(C^{0}(\bar{\Omega})\right)^{2} ; \mathbf{v}_{\mid K} \in \mathbf{P}_{1}(K) \forall K \in \mathcal{T}_{h}\right\} \cap \mathbf{M} \\
& =\left\{\mathbf{v} \in \mathbf{M} ; \mathbf{v}_{\mid K} \in \mathbf{P}_{1}(K) \forall K \in \mathcal{T}_{h}\right\} .
\end{aligned}
$$

Let us also define

$$
\mathbf{V}_{h}=K_{e r} b=\left\{\tau \in \mathbf{X}_{h} ; b(\tau, \mathbf{v})=0 \forall \mathbf{v} \in \mathbf{M}_{h}\right\} .
$$

We thus employ conforming finite elements of lowest-order ensuring that the inf-sup condition, which represents the main difficulty in the velocity-pressure formulation for the Stokes problem, is directly satisfied.

However, the bilinear form $a(\cdot, \cdot)$ is not coercive on the discrete kernel $\mathbf{V}_{h}$ of $b(\cdot, \cdot)$; cf. 4. In order to retrieve its coercivity, we apply a stabilization technique, that is we replace $a(\cdot, \cdot)$ by $a(\cdot, \cdot)+\beta A_{h}(\cdot, \cdot)$ where $\beta>0$ represents a stabilization parameter, which can be eventually chosen independently of the discretization parameter $h$.

In [4, the stabilization term $A_{h}(\cdot, \cdot)$ was defined by means of the jumps of both the pressure and the vorticity across the edges of the triangulation. In this paper we employ a simpler formulation, obtained by stabilizing only the pressure and leading to similar theoretical and numerical results.

So, we define the bilinear form $A_{h}: \mathbf{X}_{h} \times \mathbf{X}_{h} \rightarrow \mathbb{R}$ by

$$
A_{h}(\delta, \tau)=\sum_{e \in \mathcal{E}_{h} \cup \Gamma_{2}} h_{e} \int_{e}[r][q] d \Gamma, \quad \forall \delta=(\rho, r), \tau=(\theta, q) \in \mathbf{X}_{h},
$$

where $[\cdot]_{e}$ stands for the jump across the internal edge $e \in \mathcal{E}_{h}$, respectively for the trace on the edge $e$ situated on $\Gamma_{2}$. We can now consider the following discrete formulation of (2.3):

$$
\begin{cases}\text { Find } \quad\left(\sigma_{h}, \mathbf{u}_{h}\right) \in \mathbf{X}_{h} \times \mathbf{M}_{h} ; & \\ a\left(\sigma_{h}, \tau\right)+\beta A_{h}\left(\sigma_{h}, \tau\right)+b\left(\tau, \mathbf{u}_{h}\right)=0 & \forall \tau \in \mathbf{X}_{h}, \\ b\left(\sigma_{h}, \mathbf{v}\right)=l(\mathbf{v}) & \forall \mathbf{v} \in \mathbf{M}_{h} .\end{cases}
$$

It is useful to introduce the seminorm on $\mathbf{X}_{h}$ defined for all $\tau=(\theta, q) \in \mathbf{X}_{h}$ by

$$
|\tau|_{h}=\sqrt{A_{h}(\tau, \tau)}=\left(\sum_{e \in \mathcal{E}_{h} \cup \Gamma_{2}} h_{e}\|[q]\|_{0, e}^{2}\right)^{1 / 2} .
$$


We next prove that the new bilinear form $a(\cdot, \cdot)+\beta A_{h}(\cdot, \cdot)$ is $\mathbf{V}_{h^{-}}$elliptic and $\mathbf{X}_{h^{-}}$ continuous with respect to the $\mathbf{L}^{2}(\Omega)$-norm of $\mathbf{X}$, independently of $h$.

The first result is obtained by adapting the proof given in 4 for the case where the stabilization term also takes into account the vorticity. Thus one gets:

Lemma 3.1. There exists a positive constant independent of $h$ such that

$$
\|q\|_{0, \Omega} \leq c\left(\|\theta\|_{0, \Omega}+|\tau|_{h}\right), \quad \forall \tau=(\theta, q) \in \mathbf{V}_{h} .
$$

Proof. Let $\tau=(\theta, q) \in \mathbf{V}_{h}$ and let us denote by $P_{0} q=\frac{1}{|\Omega|} \int_{\Omega} q d \Omega$ and consider $\widetilde{q}=q-P_{0} q$. Then $\widetilde{q} \in L_{0}^{2}(\Omega)$ and obviously one has (cf. [13]) that :

$$
C\|\widetilde{q}\|_{0, \Omega} \leq\|\nabla q\|_{-1, \Omega}=\sup _{\mathbf{v} \in \mathbf{H}_{0}^{1}(\Omega)} \frac{\int_{\Omega} q d i v \mathbf{v} d \Omega}{|\mathbf{v}|_{1, \Omega}} .
$$

We remark for any $\mathbf{v} \in \mathbf{M} \cap \mathbf{H}^{1}(\Omega)$ and any $\mathbf{v}_{h} \in \mathbf{M}_{h}$ that

$$
\begin{aligned}
\int_{\Omega} q d i v \mathbf{v} d \Omega & =b\left(\tau, \mathbf{v}-\mathbf{v}_{h}\right)+\nu \int_{\Omega} \theta \operatorname{curl} \mathbf{v} d \Omega \\
& =\nu \int_{\Omega} \theta \operatorname{curl} \mathbf{v}_{h} d \Omega+\sum_{e \in \mathcal{E}_{h} \cup \Gamma_{2}} \int_{e}[q]\left(\mathbf{v}-\mathbf{v}_{h}\right) \cdot \mathbf{n} d \Gamma,
\end{aligned}
$$

where we have used Green's formula and the boundary conditions $\mathbf{v} \cdot \mathbf{n}=\mathbf{v}_{h} \cdot \mathbf{n}=0$ on $\Gamma_{1} \cup \Gamma_{3}$ in order to obtain the last equality. We next choose for $\mathbf{v}_{h}$ the image of $\mathbf{v}$ by a regularization operator (see [5]) with values in $\mathbf{M}_{h}$, such that :

$$
\left(\sum_{e \in \mathcal{E}_{h} \cup \Gamma_{2}} h_{e}^{-1}\left\|\left(\mathbf{v}-\mathbf{v}_{h}\right) \cdot \mathbf{n}\right\|_{0, e}^{2}\right)^{1 / 2} \leq c|\mathbf{v}|_{1, \Omega} \quad \text { and } \quad\left|\mathbf{v}_{h}\right|_{1, \Omega} \leq c|\mathbf{v}|_{1, \Omega} .
$$

Then the previous relations give

$$
\int_{\Omega} q d i v \mathbf{v} d \Omega \leq \nu\|\theta\|_{0, \Omega}\left\|\operatorname{curl} \mathbf{v}_{h}\right\|_{0, \Omega}+c|\tau|_{h}|\mathbf{v}|_{1, \Omega},
$$

which implies on the one hand,

$$
\left|\int_{\Omega} q \operatorname{div} \mathbf{v} d \Omega\right| \leq c\left(\|\theta\|_{0, \Omega}+|\tau|_{h}\right)|\mathbf{v}|_{1, \Omega}
$$

and on the other hand,

$$
\|\widetilde{q}\|_{0, \Omega} \leq c\left(\|\theta\|_{0, \Omega}+|\tau|_{h}\right) .
$$

So it is now sufficient to prove the same inequality for $P_{0} q$ in order to end the proof of the lemma. Following [4, we consider a function $\mathbf{w} \in \mathbf{M} \cap \mathbf{H}^{1}(\Omega)$ with $\int_{\Omega} d i v \mathbf{w} d \Omega=1$ and we write

$$
\begin{aligned}
\left|P_{0} q\right| & =\left|\int_{\Omega}(\widetilde{q}-q) \operatorname{div} \mathbf{w} d \Omega\right| \\
& \leq\|\widetilde{q}\|_{0, \Omega}|\mathbf{w}|_{1, \Omega}+c\left(\|\theta\|_{0, \Omega}+|\tau|_{h}\right)|\mathbf{w}|_{1, \Omega},
\end{aligned}
$$

which finally leads to the announced result.

So the uniform coercivity of $a(\cdot, \cdot)+\beta A_{h}(\cdot, \cdot)$ is given by the relation (3.3), while the uniform continuity comes from the next lemma. 
Lemma 3.2. For any $\tau=(\theta, q) \in \mathbf{X}_{h}$ one has

$$
|\tau|_{h} \leq c\|q\|_{0, \Omega}
$$

with a constant $c$ independent of $h$.

Proof. Let us recall the following well-known inequality, which holds for any $q \in$ $H^{1}(K)$, any $K \in \mathcal{T}_{h}$ and $e \subset \partial K$ :

$$
h_{e}^{-1 / 2}\|q\|_{0, e} \leq c\left(h_{K}^{-1}\|q\|_{0, K}+|q|_{1, K}\right) .
$$

Its proof is based on the trace theorem together with a passage to the reference element. Now, for a $q$ piecewise constant on every triangle, the previous relation leads to

$$
h_{e}^{1 / 2}\|[q]\|_{0, e} \leq c\left(\|q\|_{0, K_{1}}+\|q\|_{0, K_{2}}\right)
$$

for any edge $e=\partial K_{1} \cap \partial K_{2}$. By summing up on all of the edges belonging to $\mathcal{E}_{h} \cup \Gamma_{2}$ we get the desired inequality, for any $\tau=(\theta, q) \in \mathbf{X}_{h}$,

$$
|\tau|_{h}=\left(\sum_{e \in \mathcal{E}_{h} \cup \Gamma_{2}} h_{e}\|[q]\|_{0, e}^{2}\right)^{1 / 2} \leq c\left(\sum_{K \in \mathcal{T}_{h}}\|q\|_{0, K}^{2}\right)^{1 / 2}=c\|q\|_{0, \Omega} .
$$

It is also useful to establish the next result.

Lemma 3.3. With any $\tau=(\theta, q) \in \mathbf{X}_{h}$, one can associate a function $\phi_{h} \in \mathbf{H}^{1}(\Omega) \cap$ M such that

$$
\left|\phi_{h}\right|_{1, \Omega} \leq c|\tau|_{h} \quad \text { and } \quad A_{h}(\delta, \tau)=\int_{\Omega} \operatorname{rdiv} \phi_{h} d \Omega \forall \delta=(\rho, r) \in \mathbf{X}_{h}
$$

where $c$ is a constant independent of both the discretization and the stabilization parameters.

Proof. First of all, let us notice that for any $\mu \in \prod_{e \in \mathcal{E}_{h} \cup \Gamma} L^{2}(e)$ such that $\mu=0$ on any edge $e \subset \Gamma_{1} \cup \Gamma_{3}$, there exists a function $\phi_{h} \in \mathbf{H}^{1}(\Omega) \cap \mathbf{M}$, piecewise polynomial, such that

$$
\int_{e} \phi_{h} \cdot \mathbf{n} d \Gamma=\int_{e} \mu d \Gamma \quad \forall e \in \mathcal{E}_{h} \cup \Gamma
$$

and satisfying

$$
\left|\phi_{h}\right|_{1, \Omega} \leq c\left(\sum_{e \in \mathcal{E}_{h} \cup \Gamma} h_{e}^{-1}\|\mu\|_{0, e}^{2}\right)^{1 / 2} .
$$

Indeed, let us introduce the space $\mathbf{P}(K)=\operatorname{span}\left\{\lambda_{1} \lambda_{2} \mathbf{n}_{3}, \lambda_{2} \lambda_{3} \mathbf{n}_{1}, \lambda_{3} \lambda_{1} \mathbf{n}_{2}\right\} \subset$ $P_{2}(K)^{2}$ where $\left\{\lambda_{i}\right\}_{1 \leq i \leq 3}$ denotes the barycentric coordinates and $\mathbf{n}_{i}$ represents the unit outward normal vector to the edge $e_{i}$ of the triangle $K \in \mathcal{T}_{h}$. Then we construct $\phi_{h} \in\left\{\mathbf{L}^{2}(\Omega) ;\left(\phi_{h}\right)_{\mid K} \in \mathbf{P}(K) \forall K \in \mathcal{T}_{h}\right\}$ by imposing its degrees of freedom, namely $\int_{e} \phi_{h} \cdot \mathbf{n} d \Gamma=\int_{e} \mu d \Gamma$ for any edge $e \in \mathcal{E}_{h} \cup \Gamma$. Obviously, $\phi_{h}(S)=\mathbf{0}$ at any vertex $S$ of the triangulation, $\phi_{h} \cdot \mathbf{t}=0$ on any edge and moreover, $\phi_{h}$ is continuous at any midpoint of an internal edge, therefore $\phi_{h} \in \mathbf{H}^{1}(\Omega) \cap \mathbf{M}$.

We next apply this result for

$$
\mu_{\mid e}=\left\{\begin{array}{cc}
-h_{e}[q] & \text { if } e \in \mathcal{E}_{h}, \\
-h_{e} q & \text { if } e \subset \Gamma_{2}, \\
0 & \text { if } e \subset \Gamma_{1} \cup \Gamma_{3},
\end{array}\right.
$$


and we thus get that $\left|\phi_{h}\right|_{1, \Omega} \leq c|\tau|_{h}$. Moreover, for any $\delta=(\rho, r) \in \mathbf{X}_{h}$ we get that

$$
\begin{aligned}
A_{h}(\delta, \tau) & =-\sum_{e \in \mathcal{E}_{h} \cup \Gamma_{2}} \int_{e}[r] \mu d \Gamma=-\sum_{e \in \mathcal{E}_{h}} \int_{e}[r] \phi_{h} \cdot \mathbf{n} d \Gamma-\sum_{e \subset \Gamma_{2}} \int_{e} r \phi_{h} \cdot \mathbf{n} d \Gamma \\
& =\int_{\Omega} r \operatorname{riv} \phi_{h} d \Omega,
\end{aligned}
$$

which ends the proof.

We next recall from [4 the discrete inf-sup condition for the bilinear form $b(\cdot, \cdot)$ :

Lemma 3.4. The following inequality is true, with a constant $c$ independent of $h$ :

$$
\forall \mathbf{v} \in \mathbf{M}_{h}, \quad \sup _{\tau \in \mathbf{X}_{h}} \frac{b(\tau, \mathbf{v})}{\|\tau\|_{0, \Omega}} \geq c|\mathbf{v}|_{\mathbf{M}} .
$$

Proof. With any $\mathbf{v} \in \mathbf{M}_{h}$, we associate $\tau=(-\operatorname{curl} \mathbf{v}$, div $\mathbf{v}) \in \mathbf{X}_{h}$ which clearly satisfies :

$$
\begin{aligned}
b(\tau, \mathbf{v}) & =\nu\|\operatorname{curl} \mathbf{v}\|_{0, \Omega}^{2}+\|\operatorname{div} \mathbf{v}\|_{0, \Omega}^{2} \geq c|\mathbf{v}|_{\mathbf{M}}^{2}, \\
\|\tau\|_{0, \Omega} & =|\mathbf{v}|_{\mathbf{M}},
\end{aligned}
$$

so the result is obvious.

Gathering together Lemmas 3.1, 3.2 and 3.4 we see that the mixed formulation (3.2) fulfills the hypotheses of the Babuška-Brezzi theorem, uniformly with respect to $h$ (cf. for instance [6]). Hence the discrete problem (3.2) is well-posed and one can now introduce the discrete Stokes operator as follows:

$$
S_{h}: \mathbf{L}^{4 / 3}(\Omega) \rightarrow \mathbf{Y}, \quad S_{h}(\mathbf{g})=\left(\sigma_{h}, \mathbf{u}_{h}\right)
$$

where $\left(\sigma_{h}, \mathbf{u}_{h}\right) \in \mathbf{X}_{h} \times \mathbf{M}_{h}$ is the unique solution of (3.2). Obviously, $S_{h}$ is a linear and continuous operator, which satisfies the condition

$$
\forall \mathbf{g} \in \mathbf{L}^{4 / 3}(\Omega), \quad\left\|S_{h}(\mathbf{g})\right\|_{\mathbf{Y}} \leq c\|\mathbf{g}\|_{\mathbf{L}^{4 / 3}(\Omega)}
$$

with $c$ a positive constant independent of $h$ but depending on $\beta$.

Moreover, one can prove that $S_{h}$ satisfies an error bound which leads to the unconditional convergence of the approximation method. For the case of smooth data $\mathbf{g} \in \mathbf{L}^{2}(\Omega)$, one gets:

Theorem 3.5. Let $\mathbf{g} \in \mathbf{L}^{2}(\Omega)$ and let $\bar{\sigma}_{h}$ be the $\mathbf{L}^{2}(\Omega)$-projection of $\sigma$ on $\mathbf{X}_{h}$, where $(\sigma, \mathbf{u})=S(\mathbf{g})$. Then the following estimate holds:

$$
\left\|\left(S-S_{h}\right)(\mathbf{g})\right\|_{\mathbf{X} \times \mathbf{M}} \leq C\left\{h\|\mathbf{g}\|_{0, \Omega}+\left\|\sigma-\bar{\sigma}_{h}\right\|_{\mathbf{X}}+\inf _{\mathbf{v}_{h} \in \mathbf{M}_{h}}\left|\mathbf{u}-\mathbf{v}_{h}\right|_{\mathbf{M}}\right\},
$$

where $C$ is a constant independent of $h$ (but depending on $\beta$ ).

Proof. The proof is rather technical but very similar to the one presented in [4. Therefore, here we only specify the modification in the estimate of $A_{h}\left(\bar{\sigma}_{h}, \delta\right)$ for an arbitrary $\delta=(\rho, r) \in \mathbf{X}_{h}$, where $\bar{\sigma}_{h}=\left(\bar{\omega}_{h}, \bar{p}_{h}\right) \in \mathbf{X}_{h}$. For this purpose, we associate with $\delta$ the function $\phi_{h} \in \mathbf{M}$ constructed in Lemma 3.3. which clearly satisfies $\int_{\Omega} \bar{\omega}_{h}$ curl $\phi_{h} d \Omega=0$. Hence

$$
\begin{aligned}
A_{h}\left(\bar{\sigma}_{h}, \delta\right) & =b\left(\bar{\sigma}_{h}, \phi_{h}\right) \\
& =b\left(\bar{\sigma}_{h}-\sigma, \phi_{h}\right)-l\left(\phi_{h}\right)
\end{aligned}
$$

and the proof follows as in [4]. 
In the general situation of a less regular data, one can establish:

Theorem 3.6. For any $\mathbf{g} \in \mathbf{L}^{4 / 3}(\Omega)$, one has:

$$
\left\|\left(S-S_{h}\right)(\mathbf{g})\right\|_{\mathbf{Y}} \leq C\left\{\sqrt{h}\|\mathbf{g}\|_{\mathbf{L}^{4 / 3}(\Omega)}+\left\|\sigma-\bar{\sigma}_{h}\right\|_{\mathbf{X}}+\inf _{\mathbf{v}_{h} \in \mathbf{M}_{h}}\left|\mathbf{u}-\mathbf{v}_{h}\right|_{\mathbf{M}}\right\} .
$$

Proof. The proof closely follows the one of the previous theorem. The only difference appears when estimating the term

$$
\left|\int_{\Omega} \mathbf{g} \cdot \phi_{h} d \Omega\right| \leq\|\mathbf{g}\|_{\mathbf{L}^{4 / 3}(\Omega)}\left\|\phi_{h}\right\|_{\mathbf{L}^{4}(\Omega)} \leq c \sqrt{h}\|\mathbf{g}\|_{\mathbf{L}^{4 / 3}(\Omega)}\left|\phi_{h}\right|_{1, \Omega},
$$

where $\phi_{h}$ is the discrete function introduced in Theorem 3.5. see also Lemma 3.3 The last inequality is obtained in a classical way, by passing to the reference element $\widehat{K}$ and by making use of the equivalence of norms in finite dimension (see for instance [9]). Indeed, let us note that $\left|\widehat{\phi_{h}}\right|_{1, \widehat{K}}=\mathbf{0}$ implies $\widehat{\phi_{h}}=0$ on $\widehat{K}$, since $\widehat{\phi_{h}}(\widehat{S})=\mathbf{0}$ for any vertex $\widehat{S}$ of $\widehat{K}$. Therefore, one can write on every triangle $K \in \mathcal{T}_{h}$,

$$
\left\|\phi_{h}\right\|_{\mathbf{L}^{4}(K)} \leq c h^{1 / 2}\left\|\widehat{\phi_{h}}\right\|_{\mathbf{L}^{4}(\widehat{K})} \leq c h^{1 / 2}\left|\widehat{\phi}_{h}\right|_{1, \widehat{K}} \leq c h^{1 / 2}\left|\phi_{h}\right|_{1, K}
$$

which achieves the proof.

Thus, for smooth data $\mathbf{g} \in \mathbf{L}^{2}(\Omega)$ and smooth exact solution $(\sigma, \mathbf{u}) \in \mathbf{H}^{1}(\Omega) \times$ $\mathbf{H}^{2}(\Omega)$, one recovers from Theorem 3.5 that

$$
\left\|\left(S-S_{h}\right)(\mathbf{g})\right\|_{\mathbf{Y}} \leq \operatorname{ch}\|\mathbf{g}\|_{0, \Omega},
$$

that is, the approximation method for the Stokes problem has an optimal convergence rate $O(h)$.

Furthermore, Theorem 3.6 yields the unconditional convergence of the approximation, that is, the operator $S_{h}$ satisfies:

$$
\forall \mathbf{g} \in \mathbf{L}^{4 / 3}(\Omega), \quad \lim _{h \rightarrow 0}\left\|\left(S-S_{h}\right)(\mathbf{g})\right\|_{\mathbf{Y}}=0 .
$$

\section{The Discrete NAvier-Stokes Problem}

Let us now look at the numerical approximation of the Navier-Stokes problem (2.4), which we write as follows:

$$
F_{h}\left(\sigma_{h}, \mathbf{u}_{h}\right)=0 .
$$

The mapping $F_{h}$ is defined by :

$$
F_{h}: \mathbf{Y} \rightarrow \mathbf{Y}, \quad F_{h}(\tau, \mathbf{v})=(\tau, \mathbf{v})-S_{h}(\mathbf{f}-G(\tau, \mathbf{v})) .
$$

We remark that if $\left(\sigma_{h}, \mathbf{u}_{h}\right)$ is a solution of equation (4.1), then $\left(\sigma_{h}, \mathbf{u}_{h}\right) \in \mathbf{X}_{h} \times \mathbf{M}_{h}$. The functional $F_{h}$ is differentiable and for all $(\tau, \mathbf{v}) \in \mathbf{Y}$, one has:

$$
D F_{h}(\tau, \mathbf{v})=I d+S_{h} \circ D G(\tau, \mathbf{v})
$$


4.1. Existence and uniqueness. A priori error bound. The analysis of the discrete problem (4.1) uses a result mainly based on the implicit function theorem, which was first established in [7]. Some variants can be found in [15] or in [8]. In order to apply the general result of [15, we suppose that the Stokes operator $S$ satisfies the following regularity assumption:

(H2) there exists $\delta>0$ such that $S: \mathbf{L}^{4 / 3}(\Omega) \rightarrow \mathbf{H}^{\delta}(\Omega) \times \mathbf{H}^{1+\delta}(\Omega)$ is well-defined and continuous.

Remark 4.1. This last condition holds, for instance, with $\delta=1$ whenever $\Omega$ is a convex polygon and $\left|\Gamma_{2}\right|=\left|\Gamma_{3}\right|=0$; cf. [13].

As a consequence of (H2), one deduces from Theorem 3.6 that for any $\mathbf{g} \in$ $\mathbf{L}^{4 / 3}(\Omega)$,

$$
\left\|\left(S-S_{h}\right)(\mathbf{g})\right\|_{\mathbf{Y}} \leq c h^{\alpha}\|\mathbf{g}\|_{\mathbf{L}^{4 / 3}(\Omega)}
$$

where $\alpha=\min \left(\frac{1}{2}, \delta\right)$ and where $c>0$ depends on $\alpha,(\sigma, \mathbf{u})$ and $\beta$ but is independent of $h$.

Then we show, thanks to the properties (A1) and (A2) of the linear operator $S_{h}$, the following statement:

Theorem 4.2. Assume the condition (H2) holds. Then the nonlinear mapping $F_{h}$ fulfills the conditions:

(C1) there exists a positive constant $c$ independent of $h$ such that, for any $(\tau, \mathbf{v}) \in$ Y:

$$
\left\|D F_{h}(\sigma, \mathbf{u})-D F_{h}(\tau, \mathbf{v})\right\|_{\mathcal{L}(\mathbf{Y})} \leq c\|(\sigma, \mathbf{u})-(\tau, \mathbf{v})\|_{\mathbf{Y}},
$$

(C2) $\lim _{h \rightarrow 0}\left\|F_{h}(\sigma, \mathbf{u})\right\|_{\mathbf{Y}}=0$,

(C3) there exists $h_{0}>0$ such that for any $h<h_{0}, D F_{h}(\sigma, \mathbf{u})$ is an isomorphism of $\mathbf{Y}$ and

$$
\left\|D F_{h}(\sigma, \mathbf{u})^{-1}\right\|_{\mathcal{L}(\mathbf{Y})} \leq 2\left\|D F(\sigma, \mathbf{u})^{-1}\right\|_{\mathcal{L}(\mathbf{Y})}
$$

Proof. First, one has that

$$
D F_{h}(\sigma, \mathbf{u})=I d+S_{h} \circ D G(\sigma, \mathbf{u})
$$

where $D G(\sigma, \mathbf{u}): \mathbf{Y} \rightarrow \mathbf{L}^{4 / 3}(\Omega)$ is given, for any $\delta=(\rho, r) \in \mathbf{X}, \mathbf{w} \in \mathbf{L}^{4}(\Omega)$ by

$$
D G(\sigma, \mathbf{u})(\delta, \mathbf{w})=\omega \mathbf{w}^{\perp}+\rho \mathbf{u}^{\perp} .
$$

So for any $\tau=(\theta, q) \in \mathbf{X}$ and $\mathbf{v} \in \mathbf{L}^{4}(\Omega)$, the stability property (A1) together with Hölder's and Cauchy-Schwarz inequalities imply that

$$
\begin{gathered}
\left\|D F_{h}(\sigma, \mathbf{u})(\delta, \mathbf{w})-D F_{h}(\tau, \mathbf{v})(\delta, \mathbf{w})\right\|_{\mathbf{Y}} \\
\leq c\|D G(\sigma, \mathbf{u})(\delta, \mathbf{w})-D G(\tau, \mathbf{v})(\delta, \mathbf{w})\|_{\mathbf{L}^{4 / 3}(\Omega)} \\
\leq c\left(\|\omega-\theta\|_{0, \Omega}\left\|\mathbf{w}^{\perp}\right\|_{\mathbf{L}^{4}(\Omega)}+\|\rho\|_{0, \Omega}\left\|\mathbf{u}^{\perp}-\mathbf{v}^{\perp}\right\|_{\mathbf{L}^{4}(\Omega)}\right) \\
\leq c\|(\sigma, \mathbf{u})-(\tau, \mathbf{v})\|_{\mathbf{Y}}\|(\delta, \mathbf{w})\|_{\mathbf{Y}} .
\end{gathered}
$$

Therefore the condition (C1) is satisfied, with a constant $c$ independent of $h$.

Second, in order to establish the consistency property (C2) let us write

$$
\left\|F_{h}(\sigma, \mathbf{u})\right\|_{\mathbf{Y}}=\left\|F(\sigma, \mathbf{u})-F_{h}(\sigma, \mathbf{u})\right\|_{\mathbf{Y}}=\left\|\left(S-S_{h}\right)(\mathbf{f}-G(\sigma, \mathbf{u}))\right\|_{\mathbf{Y}} .
$$

So the condition (A2) immediately gives that $\lim _{h \rightarrow 0}\left\|F_{h}(\sigma, \mathbf{u})\right\|_{\mathbf{Y}}=0$. 
Third, let us show that the linear operator $D F_{h}(\sigma, \mathbf{u})$ is an isomorphism of $\mathbf{Y}$, for $h$ small enough. For this purpose, we write it as follows:

$$
D F_{h}(\sigma, \mathbf{u})=D F(\sigma, \mathbf{u}) \circ\left(I d+D F(\sigma, \mathbf{u})^{-1} \circ\left(D F_{h}(\sigma, \mathbf{u})-D F(\sigma, \mathbf{u})\right)\right) .
$$

By putting $B_{h}=D F(\sigma, \mathbf{u})^{-1} \circ\left(D F_{h}(\sigma, \mathbf{u})-D F(\sigma, \mathbf{u})\right)$, we get that

$$
D F_{h}(\sigma, \mathbf{u})=D F(\sigma, \mathbf{u}) \circ\left(I d+B_{h}\right)
$$

where $D F(\sigma, \mathbf{u})$ is invertible by hypothesis. One knows that if $\left\|B_{h}\right\|_{\mathcal{L}(\mathbf{Y})}<1$, then $D F_{h}(\sigma, \mathbf{u})$ is an isomorphism and moreover, the next bound holds:

$$
\left\|D F_{h}(\sigma, \mathbf{u})^{-1}\right\|_{\mathcal{L}(\mathbf{Y})} \leq \frac{\left\|D F(\sigma, \mathbf{u})^{-1}\right\|_{\mathcal{L}(\mathbf{Y})}}{1-\left\|B_{h}\right\|_{\mathcal{L}(\mathbf{Y})}}
$$

So it is now sufficient to prove that $\left\|B_{h}\right\|_{\mathcal{L}(\mathbf{Y})}<\frac{1}{2}$ for $h$ small enough, in order to establish the stability property (C3). Let us note that

$$
\left\|B_{h}\right\|_{\mathcal{L}(\mathbf{Y})} \leq\left\|D F(\sigma, \mathbf{u})^{-1}\right\|_{\mathcal{L}(\mathbf{Y})}\left\|\left(S-S_{h}\right) \circ D G(\sigma, \mathbf{u})\right\|_{\mathcal{L}(\mathbf{Y})} .
$$

Thanks to the supposed regularity (H2) of the associated Stokes problem, one has that

$$
\forall \mathbf{g} \in \mathbf{L}^{4 / 3}(\Omega), \quad\left\|\left(S-S_{h}\right)(\mathbf{g})\right\|_{\mathbf{Y}} \leq c h^{\alpha}\|\mathbf{g}\|_{\mathbf{L}^{4 / 3}(\Omega)}
$$

Since $D G(\sigma, \mathbf{u})$ is a bounded operator from $\mathbf{Y}$ to $\mathbf{L}^{4 / 3}(\Omega)$, we get that

$$
\lim _{h \rightarrow 0}\left\|\left(S-S_{h}\right) \circ D G(\sigma, \mathbf{u})\right\|_{\mathcal{L}(\mathbf{Y})}=0
$$

which yields $\left\|B_{h}\right\|_{\mathcal{L}(\mathbf{Y})}<\frac{1}{2}$ for $h$ smaller than a given $h_{0}>0$. This achieves the proof.

Then the next statement is true, according to [15].

Theorem 4.3. Assume (H2). Then there exist $h_{1}>0$ and $\delta>0$ such that, for all $h<h_{1}$, problem (4.1) has a unique solution satisfying $\left\|(\sigma, \mathbf{u})-\left(\sigma_{h}, \mathbf{u}_{h}\right)\right\|_{\mathbf{Y}} \leq \delta$. Moreover, the following a priori, respectively a posteriori estimates hold:

$$
\begin{aligned}
&\left\|(\sigma, \mathbf{u})-\left(\sigma_{h}, \mathbf{u}_{h}\right)\right\|_{\mathbf{Y}} \leq c\left\|F_{h}(\sigma, \mathbf{u})\right\|_{\mathbf{Y}}, \\
&\left\|(\sigma, \mathbf{u})-\left(\sigma_{h}, \mathbf{u}_{h}\right)\right\|_{\mathbf{Y}} \leq c^{\prime}\left\|F\left(\sigma_{h}, \mathbf{u}_{h}\right)\right\|_{\mathbf{Y}}
\end{aligned}
$$

with $c, c^{\prime}$ independent of the discretization.

Therefore, the approximation method for the Navier-Stokes problem is unconditionally convergent thanks to the condition (C2). Moreover, its convergence rate is given by an upper bound for

$$
\left\|F_{h}(\sigma, \mathbf{u})\right\|_{\mathbf{Y}}=\left\|\left(S-S_{h}\right)(\mathbf{f}-G(\sigma, \mathbf{u}))\right\|_{\mathbf{Y}} ;
$$

see Theorem 3.6 .

When considering a smooth data $\mathbf{f} \in \mathbf{L}^{2}(\Omega)$ and assuming that the exact solution $(\sigma, \mathbf{u})$ of the initial Navier-Stokes problem satisfies $(\sigma, \mathbf{u}) \in \mathbf{H}^{1}(\Omega) \times \mathbf{H}^{2}(\Omega)$, we see, on the one hand, that $\omega \in L^{4}(\Omega)$ so $G(\sigma, \mathbf{u}) \in \mathbf{L}^{2}(\Omega)$ and

$$
\|G(\sigma, \mathbf{u})\|_{0, \Omega} \leq\|\omega\|_{L^{4}(\Omega)}\left\|\mathbf{u}^{\perp}\right\|_{\mathbf{L}^{4}(\Omega)} \leq c|\sigma|_{1, \Omega}\|\mathbf{u}\|_{1, \Omega} .
$$

On the other hand, Theorem 3.5 gives that

$$
\begin{aligned}
\left\|(\sigma, \mathbf{u})-\left(\sigma_{h}, \mathbf{u}_{h}\right)\right\|_{\mathbf{Y}} & \leq c\left\|\left(S-S_{h}\right)(\mathbf{f}-G(\sigma, \mathbf{u}))\right\|_{\mathbf{Y}} \\
& \leq \operatorname{ch}\left\{\|\mathbf{f}-G(\sigma, \mathbf{u})\|_{0, \Omega}+|\sigma|_{1, \Omega}+|\mathbf{u}|_{2, \Omega}\right\}
\end{aligned}
$$


so we obtain a convergence rate $O(h)$ for the $\mathbf{Y}$-norm of the error, as for the Stokes problem.

Remark 4.4. By means of a technical argument of Aubin-Nitsche type, one can improve the convergence rate for the velocity in the $\mathbf{L}^{4}(\Omega)$-norm and obtain $O\left(h^{3 / 2}\right)$, whenever $\mathbf{f} \in \mathbf{L}^{2}(\Omega)$ and $(\sigma, \mathbf{u}) \in \mathbf{H}^{1}(\Omega) \times \mathbf{H}^{2}(\Omega)$. A detailed proof, including the case of nonhomogeneous boundary conditions $p_{0}$ and $\omega_{0}$, can be found in [2]. So, the proposed approximation method for the Navier-Stokes problem is optimal in terms of finite elements.

4.2. A posteriori error estimate. Theorem 4.3 says that an a posteriori error indicator is defined by an upper bound for $\left\|F\left(\sigma_{h}, \mathbf{u}_{h}\right)\right\|_{\mathbf{Y}}$. For this purpose we estimate, for any $\tau=(\theta, q) \in \mathbf{X}$ and any $\mathbf{v} \in \mathbf{M}$, the quantity $\left\langle F\left(\sigma_{h}, \mathbf{u}_{h}\right),(\tau, \mathbf{v})\right\rangle$ where $\langle\cdot, \cdot\rangle$ now denotes the scalar product of $\mathbf{X} \times \mathbf{M}$. We clearly have, since $F_{h}\left(\sigma_{h}, \mathbf{u}_{h}\right)=0$, that

$$
\left\langle F\left(\sigma_{h}, \mathbf{u}_{h}\right),(\tau, \mathbf{v})\right\rangle=\left\langle F\left(\sigma_{h}, \mathbf{u}_{h}\right),(\tau, \mathbf{v})\right\rangle-\left\langle F_{h}\left(\sigma_{h}, \mathbf{u}_{h}\right),\left(\tau_{h}, \mathbf{v}_{h}\right)\right\rangle
$$

for any $\tau_{h}=\left(\theta_{h}, q_{h}\right) \in \mathbf{X}_{h}, \mathbf{v}_{h} \in \mathbf{M}_{h}$. So, taking into account the definition of the mappings $F$ and $F_{h}$, we can write

$$
\begin{aligned}
\left\langle F\left(\sigma_{h}, \mathbf{u}_{h}\right),(\tau, \mathbf{v})\right\rangle= & \nu \int_{\Omega} \omega_{h}\left(\theta-\theta_{h}\right) d \Omega-\beta \sum_{e \in E_{h} \cup \Gamma_{2}} h_{e} \int_{e}\left[p_{h}\right]\left[q_{h}\right] d \Gamma \\
& -\nu \int_{\Omega}\left(\theta-\theta_{h}\right) \operatorname{curl} \mathbf{u}_{h} d \Omega+\int_{\Omega}\left(q-q_{h}\right) \operatorname{div} \mathbf{u}_{h} d \Omega \\
& +\nu \int_{\Omega} \omega_{h} \operatorname{curl}\left(\mathbf{v}-\mathbf{v}_{h}\right) d \Omega-\int_{\Omega} p_{h} \operatorname{div}\left(\mathbf{v}-\mathbf{v}_{h}\right) d \Omega \\
& -\int_{\Omega}\left(\mathbf{f}-\omega_{h} \mathbf{u}_{h}^{\perp}\right) \cdot\left(\mathbf{v}-\mathbf{v}_{h}\right) d \Omega .
\end{aligned}
$$

In what follows, we take $\tau_{h}=(0,0)$ and $\mathbf{v}_{h}=R_{h} \mathbf{v}$ where $R_{h}$ denotes the Clément's interpolation operator. Next, we integrate by parts on every $K \in \mathcal{T}_{h}$ and we take into account that $\operatorname{curl} \omega_{h}=0$ and $\nabla p_{h}=0$ on every triangle $K$. Then finally we get:

$$
\begin{aligned}
\left\langle F\left(\sigma_{h}, \mathbf{u}_{h}\right),(\tau, \mathbf{v})\right\rangle= & \nu \int_{\Omega}\left(\omega_{h}-\operatorname{curl} \mathbf{u}_{h}\right) \theta d \Omega+\nu \sum_{e \in \mathcal{E}_{h}} \int_{e}\left[\omega_{h}\right]\left(\mathbf{v}-R_{h} \mathbf{v}\right) \cdot \mathbf{t} d \Gamma \\
& -\int_{\Omega}\left(\mathbf{f}-\omega_{h} \mathbf{u}_{h}^{\perp}\right) \cdot\left(\mathbf{v}-R_{h} \mathbf{v}\right) d \Omega+\int_{\Omega} q d i v \mathbf{u}_{h} d \Omega \\
& -\sum_{e \subset \Gamma_{2}} \int_{e} p_{h}\left(\mathbf{v}-R_{h} \mathbf{v}\right) \cdot \mathbf{n} d \Gamma+\nu \sum_{e \subset \Gamma_{3}} \int_{e} \omega_{h}\left(\mathbf{v}-R_{h} \mathbf{v}\right) \cdot \mathbf{t} d \Gamma \\
& -\sum_{e \in \mathcal{E}_{h}} \int_{e}\left[p_{h}\right]\left(\mathbf{v}-R_{h} \mathbf{v}\right) \cdot \mathbf{n} d \Gamma .
\end{aligned}
$$

Let us introduce the following residuals, on every triangle $K$ of the triangulation,

$$
\begin{aligned}
& \eta_{1}=\nu\left(\omega_{h}-\operatorname{curl} \mathbf{u}_{h}\right), \\
& \eta_{2}=\operatorname{div} \mathbf{u}_{h}, \\
& \eta_{3}=\mathbf{f}-\omega_{h} \mathbf{u}_{h}^{\perp}
\end{aligned}
$$


respectively on every edge $e$ :

$$
\eta_{4}=\left\{\begin{array}{cc}
\nu\left[\omega_{h}\right] & \text { if } e \in E_{h}, \\
\nu \omega_{h} & \text { if } e \subset \Gamma_{3}, \\
0 & \text { if } e \subset \Gamma_{1} \cup \Gamma_{2},
\end{array} \quad \eta_{5}=\left\{\begin{array}{cc}
{\left[p_{h}\right]} & \text { if } e \in E_{h}, \\
p_{h} & \text { if } e \subset \Gamma_{2}, \\
0 & \text { if } e \subset \Gamma_{1} \cup \Gamma_{3} .
\end{array}\right.\right.
$$

Then one can write that

$$
\begin{aligned}
\left\langle F\left(\sigma_{h}, \mathbf{u}_{h}\right),(\tau, \mathbf{v})\right\rangle= & \sum_{K \in \mathcal{T}_{h}}\left(\int_{K} \eta_{1} \theta d \Omega+\int_{K} \eta_{2} q d \Omega-\int_{K} \eta_{3} \cdot\left(\mathbf{v}-R_{h} \mathbf{v}\right) d \Omega\right) \\
& +\sum_{e \in \mathcal{E}_{h} \cup \Gamma}\left(\int_{e} \eta_{4}\left(\mathbf{v}-R_{h} \mathbf{v}\right) \cdot \mathbf{t} d \Gamma-\int_{e} \eta_{5}\left(\mathbf{v}-R_{h} \mathbf{v}\right) \cdot \mathbf{n} d \Gamma\right) .
\end{aligned}
$$

We use the following interpolation results for $\mathbf{v} \in \mathbf{M} \subset \mathbf{H}^{s}(\Omega)$ with $\left.\left.s \in\right] 1 / 2,1\right]$ (cf. for instance [5] or [13]):

$$
\begin{aligned}
\left\|\mathbf{v}-R_{h} \mathbf{v}\right\|_{0, K} & \leq c h_{K}^{s} \sum_{K^{\prime} \in S_{K}}|\mathbf{v}|_{s, K^{\prime}}, \\
\left\|\mathbf{v}-R_{h} \mathbf{v}\right\|_{0, e} & \leq c h_{e}^{1 / 2} h_{K}^{s-1} \sum_{K^{\prime} \in S_{K}}|\mathbf{v}|_{s, K^{\prime}}
\end{aligned}
$$

where $e$ is an edge of $K$ and $S_{K}$ is the set of triangles $K^{\prime}$ such that $K \cap K^{\prime} \neq \emptyset$. This finally leads to

$$
\left|\left\langle F\left(\sigma_{h}, \mathbf{u}_{h}\right),(\tau, \mathbf{v})\right\rangle\right| \leq C\left(\|\theta\|_{0, \Omega}+\|q\|_{0, \Omega}+|\mathbf{v}|_{\mathbf{M}}\right)\left(\sum_{K \in \mathcal{T}_{h}} \eta(K)^{2}\right)^{1 / 2},
$$

where the local indicator $\eta(K)$ is defined on every triangle $K$ by

$$
\eta(K)^{2}=\left\|\eta_{1}\right\|_{0, K}^{2}+\left\|\eta_{2}\right\|_{0, K}^{2}+h_{K}^{2 s}\left\|\eta_{3}\right\|_{0, K}^{2}+h_{K}^{2 s-2} \sum_{e \in \partial K} h_{e}\left(\left\|\eta_{4}\right\|_{0, e}^{2}+\left\|\eta_{5}\right\|_{0, e}^{2}\right) .
$$

To conclude this section, let us point out that the previous estimate together with Theorem 4.3 lead to:

Theorem 4.5. Under the hypotheses of Theorem 4.3, the following a posteriori error bound holds, with a constant $C$ independent of the triangulation and of the stabilization parameter:

$$
\left\|(\sigma, \mathbf{u})-\left(\sigma_{h}, \mathbf{u}_{h}\right)\right\|_{\mathbf{Y}} \leq C\left(\sum_{K \in \mathcal{T}_{h}} \eta(K)^{2}\right)^{1 / 2}
$$

\section{NumericAl RESUlts}

We begin this section by presenting some numerical examples which illustrate the theoretical results obtained in the previous sections. More realistic cases which are usually considered for the Navier-Stokes equations are treated next.

5.1. Convergence rate. The considered domain is $\Omega=]-1,1[2$ while the exact solution is

$$
\begin{gathered}
u_{1}=\sin (\pi(x+1) / 2) \cos (\pi(y+1) / 2), \\
u_{2}=\cos (\pi(x+1) / 2) \sin (\pi(y+1) / 2), \\
p=\sin (\pi(x+1) / 2) \sin (\pi(y+1) / 2) .
\end{gathered}
$$


We impose the pressure and the vorticity respectively on the left and right boundaries whereas the velocity is given on the other boundaries. The nonlinear system is solved by a Newton algorithm.

We next compare the numerical errors obtained for different values of the parameters $h$ and $\beta$ and for Reynolds numbers varying from 1 to 10000. In Table 1 to Table 5 we present the error in the $L^{2}$-norm for the pressure and for the vorticity while the error for the velocity is given in both $L^{4}$ and $H^{1}$-norms.

One may notice that the errors on $p$ and $\omega$ are both in $O(h)$ and the error on $\mathbf{u}$ is in $O\left(h^{2}\right)$ in the $L^{4}$-norm and in $O(h)$ in the $H^{1}$-norm.

In [2], we have also computed for the above example the a posteriori estimator and applied it in order to locally refine the mesh, for $R e=1$ and $R e=1000$. The comparison with the exact error highlights the good local behaviour of the a posteriori indicator.

TABLE 1. Errors for $R e=1$ and $\beta=0.2$

\begin{tabular}{|c|c|c|c|c|}
\hline & $8 \times 8$ & $16 \times 16$ & $32 \times 32$ & $64 \times 64$ \\
\hline \hline$\left\|\omega-\omega_{h}\right\|_{L^{2}(\Omega)}$ & 0.082 & 0.038 & 0.0184 & 0.0086 \\
\hline$\left\|p-p_{h}\right\|_{L^{2}(\Omega)}$ & 0.12 & 0.053 & 0.020 & 0.009 \\
\hline$\left|u_{1}-u_{1 h}\right|_{H^{1}(\Omega)}$ & 0.17 & 0.084 & 0.041 & 0.02 \\
\hline$\left|u_{2}-u_{2 h}\right|_{H^{1}(\Omega)}$ & 0.16 & 0.081 & 0.040 & 0.02 \\
\hline$\left\|u_{1}-u_{1 h}\right\|_{L^{4}(\Omega)}$ & 0.020 & 0.006 & 0.0016 & 0.00047 \\
\hline$\left\|u_{2}-u_{2 h}\right\|_{L^{4}(\Omega)}$ & 0.026 & 0.0062 & 0.0016 & 0.00047 \\
\hline
\end{tabular}

TABle 2. Errors for $R e=10$ and $\beta=0.5$

\begin{tabular}{|c|c|c|c|c|}
\hline & $8 \times 8$ & $16 \times 16$ & $32 \times 32$ & $64 \times 64$ \\
\hline \hline$\left\|\omega-\omega_{h}\right\|_{L^{2}(\Omega)}$ & 0.090 & 0.039 & 0.0185 & 0.0087 \\
\hline$\left\|p-p_{h}\right\|_{L^{2}(\Omega)}$ & 0.074 & 0.027 & 0.0119 & 0.0056 \\
\hline$\left|u_{1}-u_{1 h}\right|_{H^{1}(\Omega)}$ & 0.178 & 0.084 & 0.041 & 0.02 \\
\hline$\left|u_{2}-u_{2 h}\right|_{H^{1}(\Omega)}$ & 0.166 & 0.081 & 0.040 & 0.02 \\
\hline$\left\|u_{1}-u_{1 h}\right\|_{L^{2}(\Omega)}$ & 0.023 & 0.0062 & 0.0016 & 0.00047 \\
\hline$\left\|u_{2}-u_{2 h}\right\|_{L^{2}(\Omega)}$ & 0.027 & 0.0065 & 0.0017 & 0.00047 \\
\hline
\end{tabular}

5.2. Realistic cases. In what follows, we present the cavity test, the step test and the T-shaped domain test. For all these classical examples, we have employed the same adaptive mesh technique based on the a posteriori estimator, but we have applied it in this paper only for the step test and the T-shaped domain. More precisely, the solution and the estimator are first computed on a nonrefined mesh and then we adapt the mesh and recalculate the approximation. Finally, the a posteriori error is calculated again on the fine mesh in order to illustrate the improvement of the solution.

We next show the velocity, pressure and vorticity obtained by this method. The numerical tests were carried out for several Reynolds numbers, however here we present only the most significant results. 
TABLE 3. Errors for $R e=100$ and $\beta=1$

\begin{tabular}{|c|c|c|c|c|}
\hline & $8 \times 8$ & $16 \times 16$ & $32 \times 32$ & $64 \times 64$ \\
\hline \hline$\left\|\omega-\omega_{h}\right\|_{L^{2}(\Omega)}$ & 0.098 & 0.042 & 0.019 & 0.009 \\
\hline$\left\|p-p_{h}\right\|_{L^{2}(\Omega)}$ & 0.068 & 0.026 & 0.011 & 0.005 \\
\hline$\left|u_{1}-u_{1 h}\right|_{H^{1}(\Omega)}$ & 0.18 & 0.085 & 0.041 & 0.02 \\
\hline$\left|u_{2}-u_{2 h}\right|_{H^{1}(\Omega)}$ & 0.17 & 0.083 & 0.041 & 0.02 \\
\hline$\left\|u_{1}-u_{1 h}\right\|_{L^{4}(\Omega)}$ & 0.025 & 0.0077 & 0.0022 & 0.0007 \\
\hline$\left\|u_{2}-u_{2 h}\right\|_{L^{4}(\Omega)}$ & 0.026 & 0.0078 & 0.0023 & 0.0007 \\
\hline
\end{tabular}

TABLE 4. Errors for $R e=1000$ and $\beta=1.5$

\begin{tabular}{|c|c|c|c|c|}
\hline & $8 \times 8$ & $16 \times 16$ & $32 \times 32$ & $64 \times 64$ \\
\hline \hline$\left\|\omega-\omega_{h}\right\|_{L^{2}(\Omega)}$ & 0.12 & 0.054 & 0.024 & 0.012 \\
\hline$\left\|p-p_{h}\right\|_{L^{2}(\Omega)}$ & 0.065 & 0.026 & 0.012 & 0.006 \\
\hline$\left|u_{1}-u_{1 h}\right|_{H^{1}(\Omega)}$ & 0.18 & 0.086 & 0.042 & 0.021 \\
\hline$\left|u_{2}-u_{2 h}\right|_{H^{1}(\Omega)}$ & 0.18 & 0.087 & 0.042 & 0.021 \\
\hline$\left\|u_{1}-u_{1 h}\right\|_{L^{4}(\Omega)}$ & 0.027 & 0.0088 & 0.0025 & 0.0008 \\
\hline$\left\|u_{2}-u_{2 h}\right\|_{L^{4}(\Omega)}$ & 0.028 & 0.0089 & 0.0025 & 0.0008 \\
\hline
\end{tabular}

TABLE 5. Errors for $R e=10000$ and $\beta=1.5$

\begin{tabular}{|c|c|c|c|c|}
\hline & $8 \times 8$ & $16 \times 16$ & $32 \times 32$ & $64 \times 64$ \\
\hline \hline$\left\|\omega-\omega_{h}\right\|_{L^{2}(\Omega)}$ & - & 0.065 & 0.037 & 0.019 \\
\hline$\left\|p-p_{h}\right\|_{L^{2}(\Omega)}$ & - & 0.029 & 0.012 & 0.005 \\
\hline$\left|u_{1}-u_{1 h}\right|_{H^{1}(\Omega)}$ & - & 0.093 & 0.046 & 0.023 \\
\hline$\left|u_{2}-u_{2 h}\right|_{H^{1}(\Omega)}$ & - & 0.093 & 0.046 & 0.023 \\
\hline$\left\|u_{1}-u_{1 h}\right\|_{L^{4}(\Omega)}$ & - & 0.015 & 0.004 & 0.0012 \\
\hline$\left\|u_{2}-u_{2 h}\right\|_{L^{4}(\Omega)}$ & - & 0.015 & 0.004 & 0.0012 \\
\hline
\end{tabular}

Cavity domain. Here we consider two different cavity tests depending on the size of the domain. We impose the velocity on the whole boundary. We take $\mathbf{u}$ equal to $(1,0)$ on the upper boundary and equal to zero on the rest of the boundary. In the first example, the domain is the unit square and the considered Reynolds number is 500 (see Figure 5.1).

In the second example, the domain is the rectangle $] 0,1[\times] 0,2[$, and we take two Reynolds numbers, respectively equal to 5000 (see Figure 5.2) and to 20000 (see Figure 5.3) . One can notice that for big Reynolds numbers, a second vortex appears in the lower part of the domain (cf. Figure 5.3). 

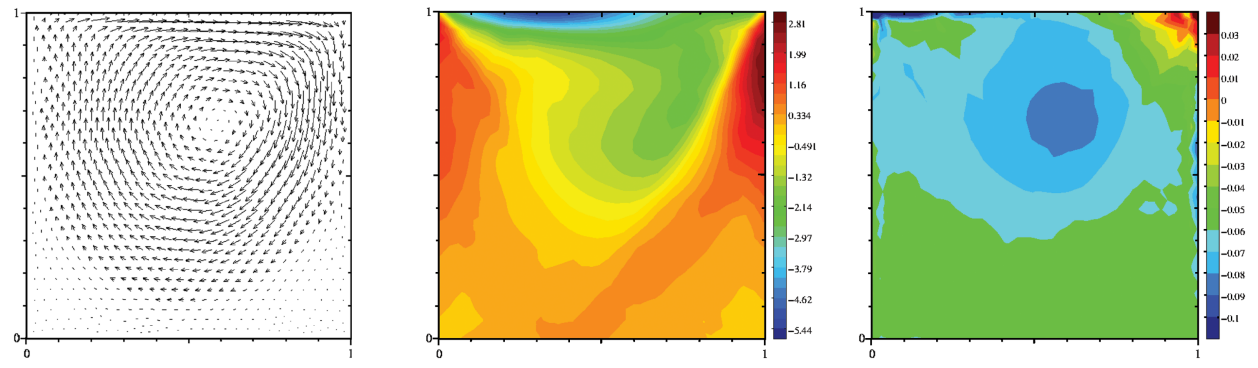

Figure 5.1. First cavity test: Velocity, vorticity and pressure $(R e=500)$
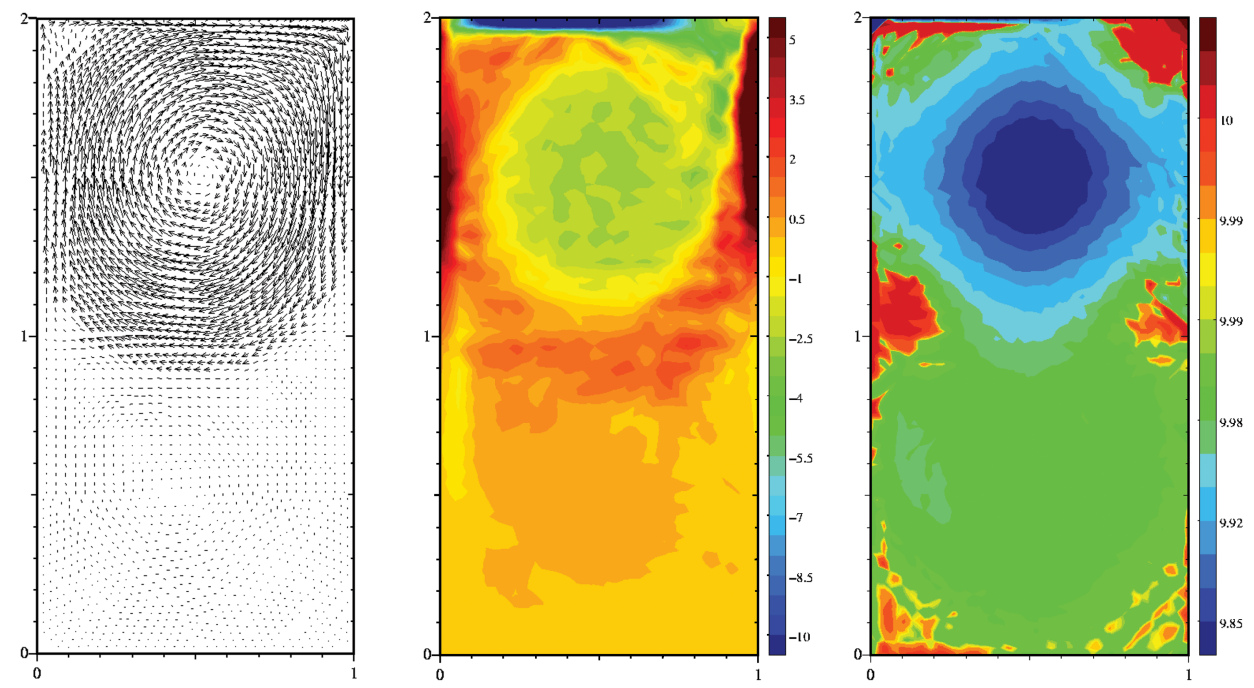

Figure 5.2. Second cavity test: Velocity, vorticity and pressure $(R e=5000)$
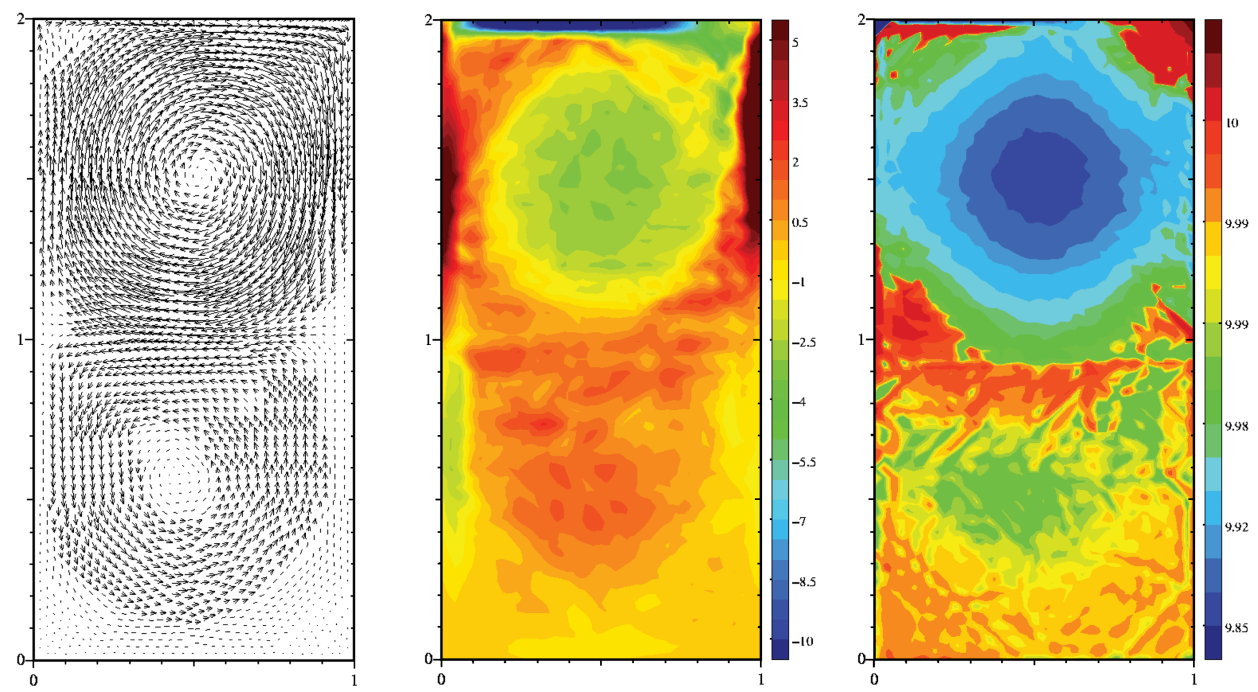

FiguRE 5.3. Second cavity test: Velocity, vorticity and pressure $(R e=20000)$ 
Step test. We are now interested in the classical step test, with the pressure given on the inlet and outlet boundaries and a zero velocity imposed elsewhere. Similar results were obtained when taking $\omega=0$ and $\mathbf{u . n}=0$ on the upper boundary but they are not presented here. For a small Reynolds number (for instance $R e=10$ ), we retrieve a linear pressure (cf. Figure [5.9) and a laminar flow (see Figures 5.7] 5.8), which is no longer the case for $R e=1000$ (Figures 5.10, 5.11 and 5.12). One may also use the a posteriori estimator in order to optimally choose the stabilization parameter $\beta$ when the Reynolds number is fixed (for instance, for the three values of $\beta$ employed in Figures [5.4, [5.5] and 5.6] one can see that $\beta=0.5$ leads to the smallest error indicator).

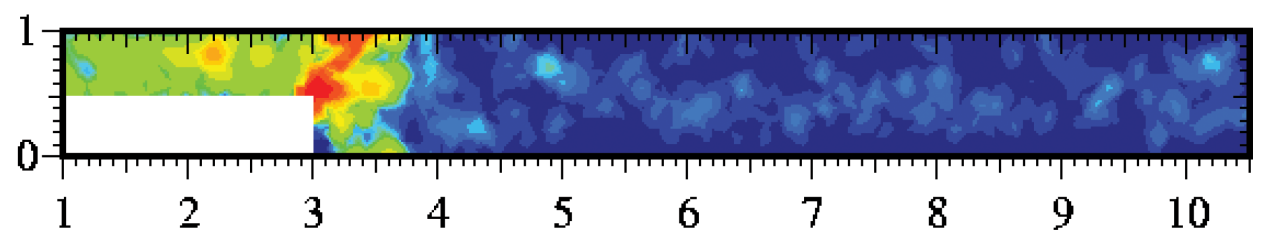

Figure 5.4. Step test: Estimator $(R e=10$ and $\beta=0.03)$

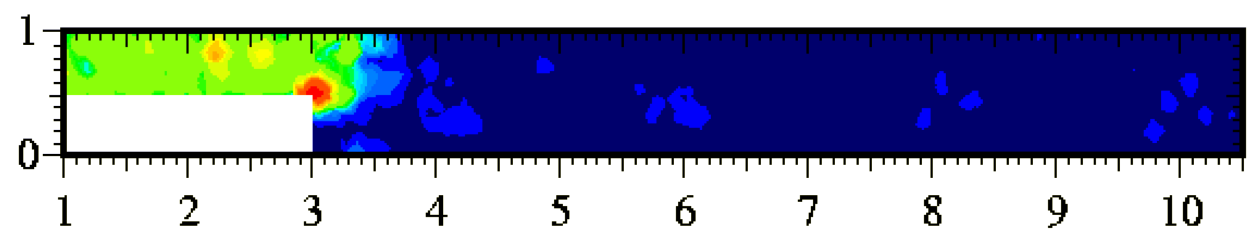

Figure 5.5. Step test: Estimator $(R e=10$ and $\beta=0.5)$
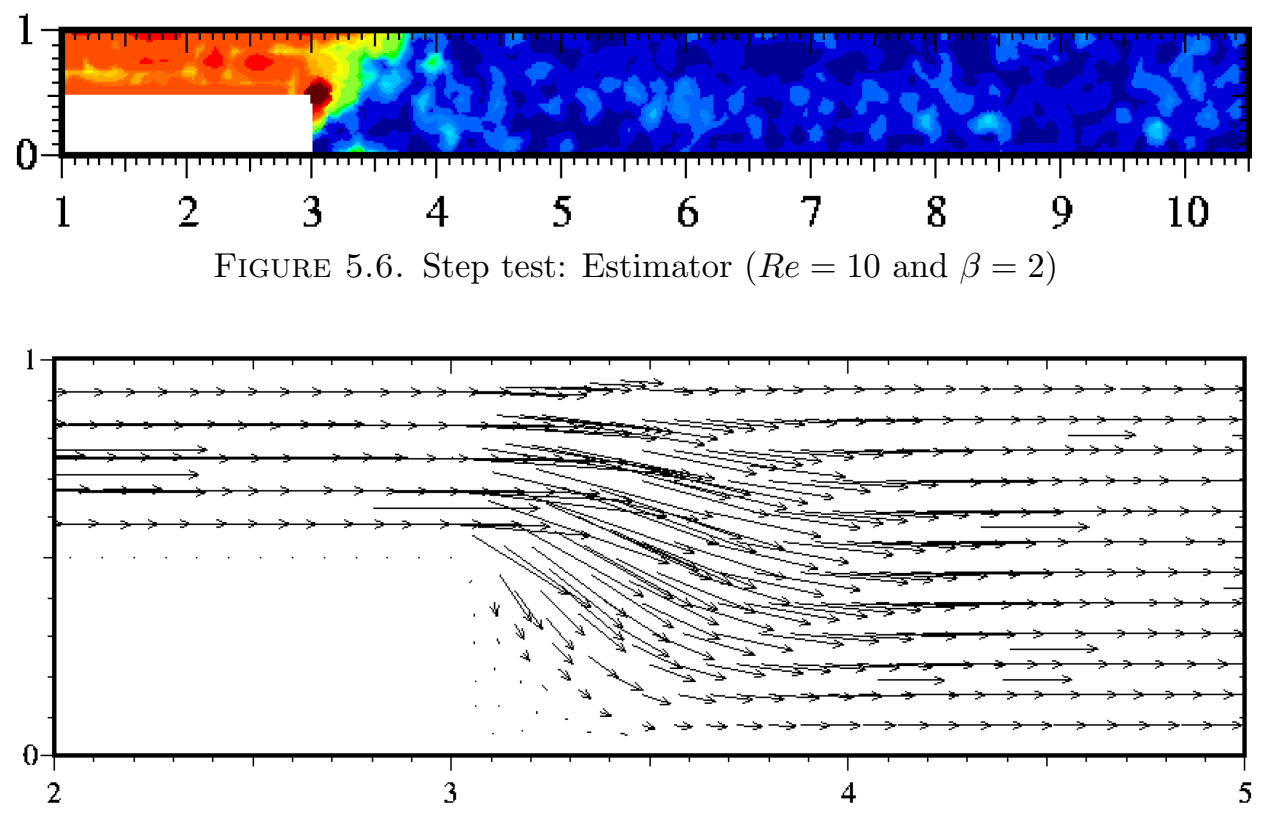

Figure 5.7. Step test: Velocity near the step $(R e=10)$ 

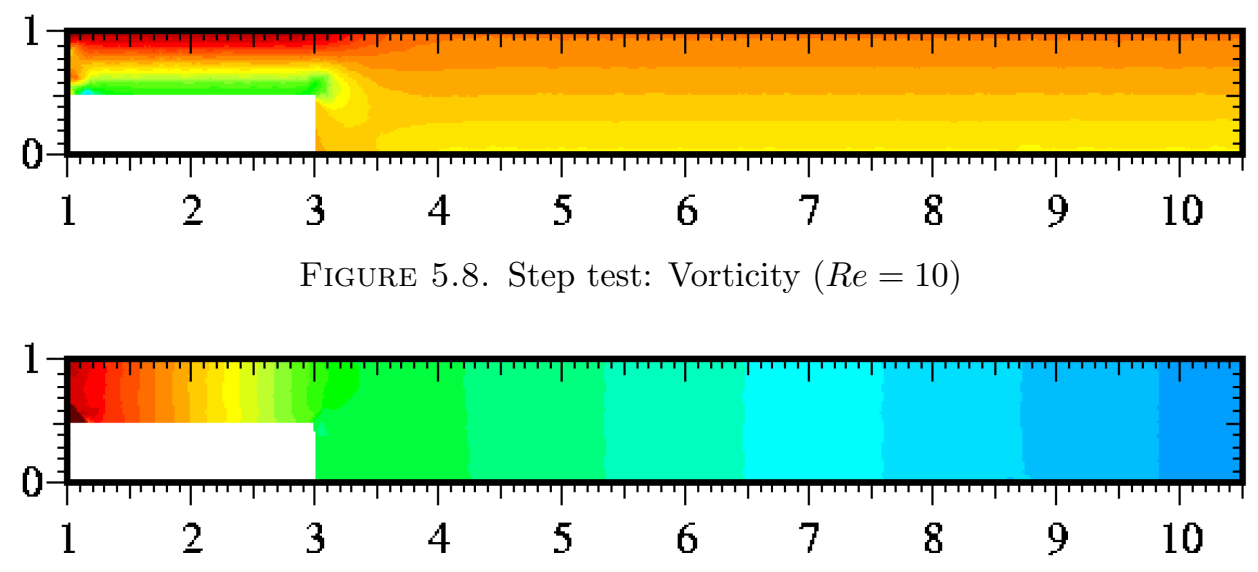

Figure 5.9. Step test: Pressure $(R e=10)$

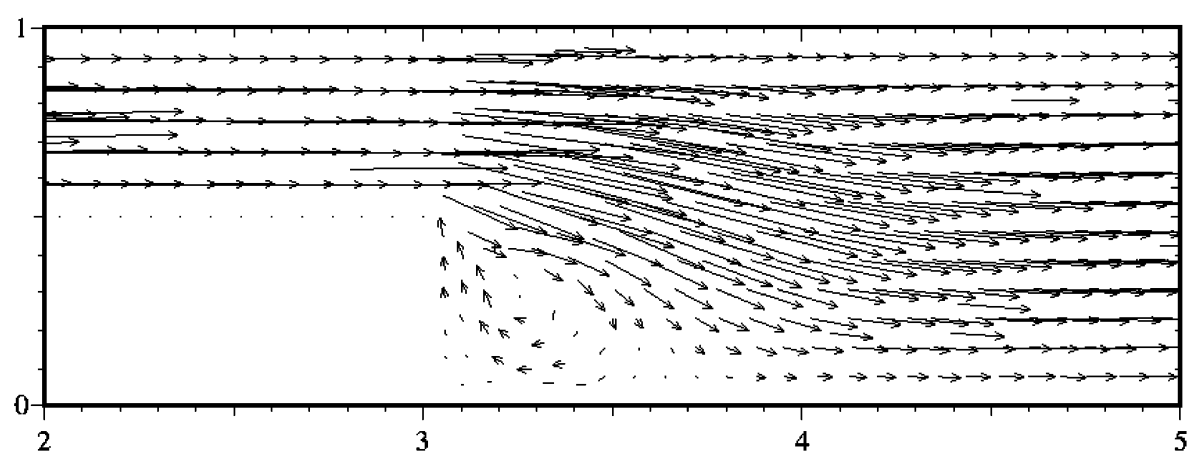

Figure 5.10. Step test: Velocity near the step $(R e=1000)$

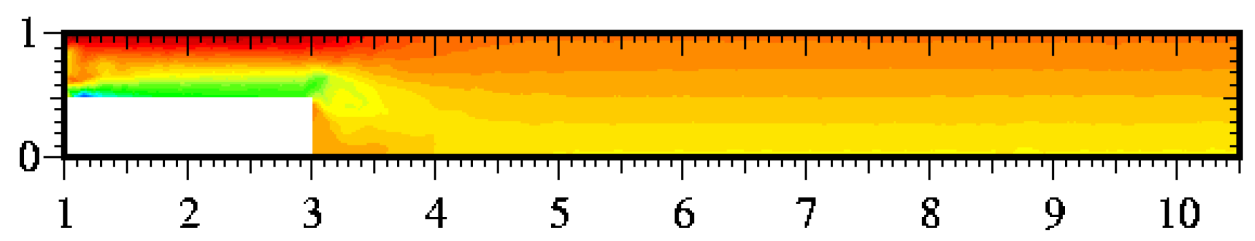

Figure 5.11. Step test: Vorticity $(R e=1000)$

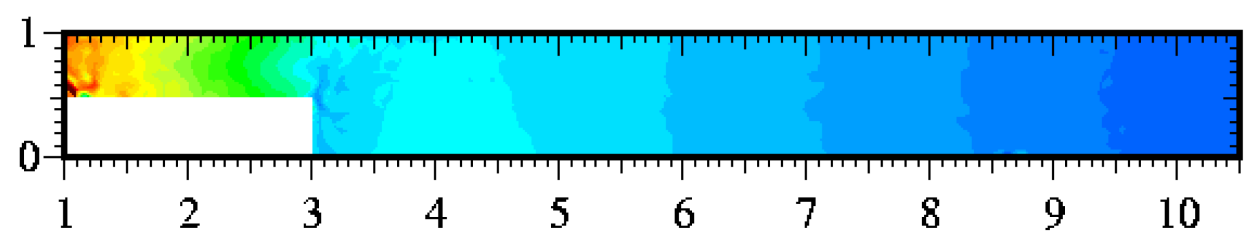

Figure 5.12. Step test: Pressure $(R e=1000)$ 
T-shaped domain. To end this section, numerical tests validating the method are also carried out on a T-shaped domain with the same boundary conditions as in the example of the previous step. Here we present the error estimator on the initial and refined grid, as well as the velocity, vorticity and pressure on the refined mesh for two different Reynolds numbers, $R e=100$ and $R e=10000$.

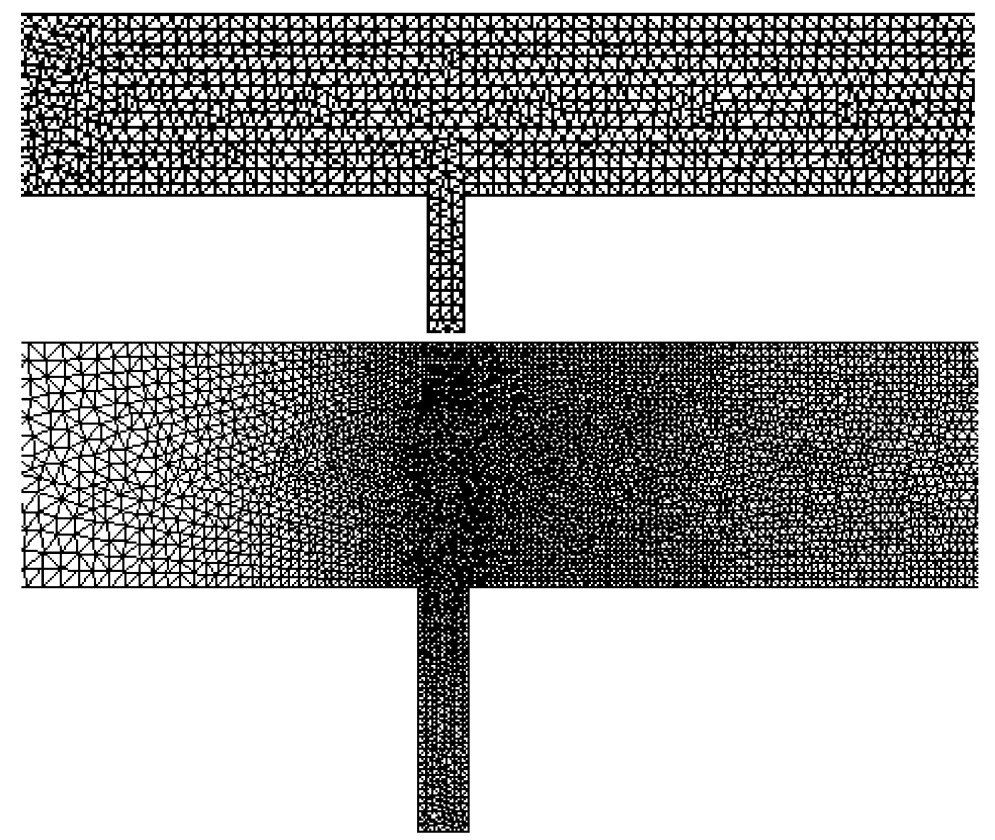

FiguRE 5.13. T-shaped test: Initial and refined grids

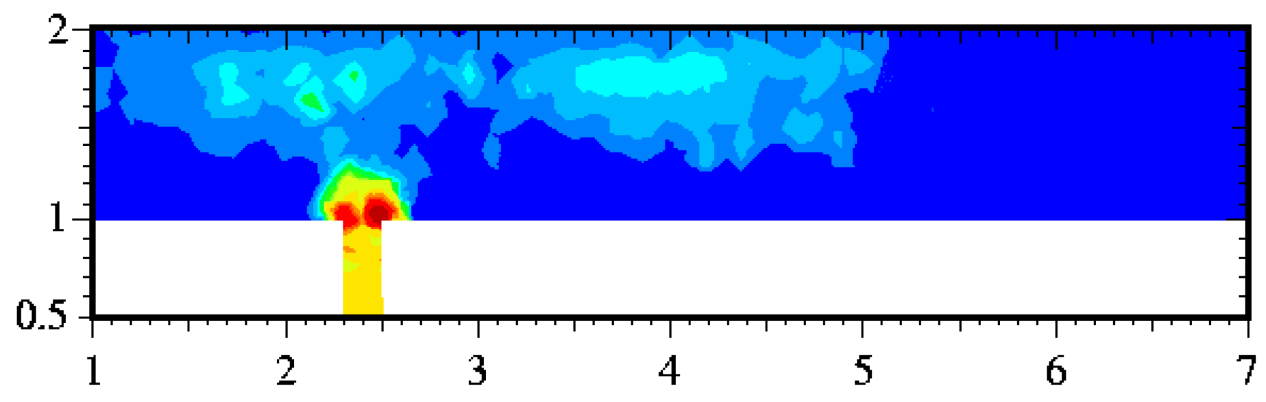

Figure 5.14. T-shaped test: Estimator on the initial grid $(R e=100)$ 


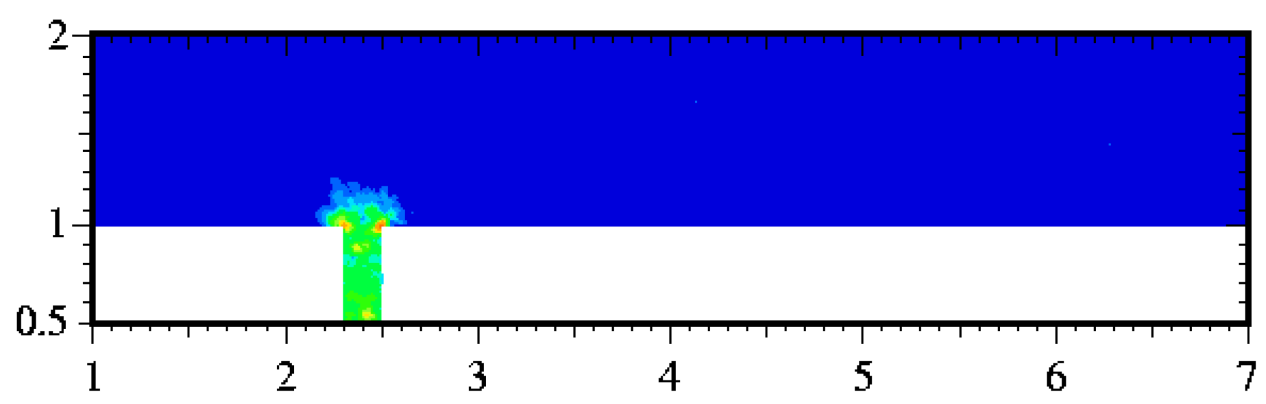

FIGURE 5.15. T-shaped test: Estimator on the refined grid $(R e=100)$
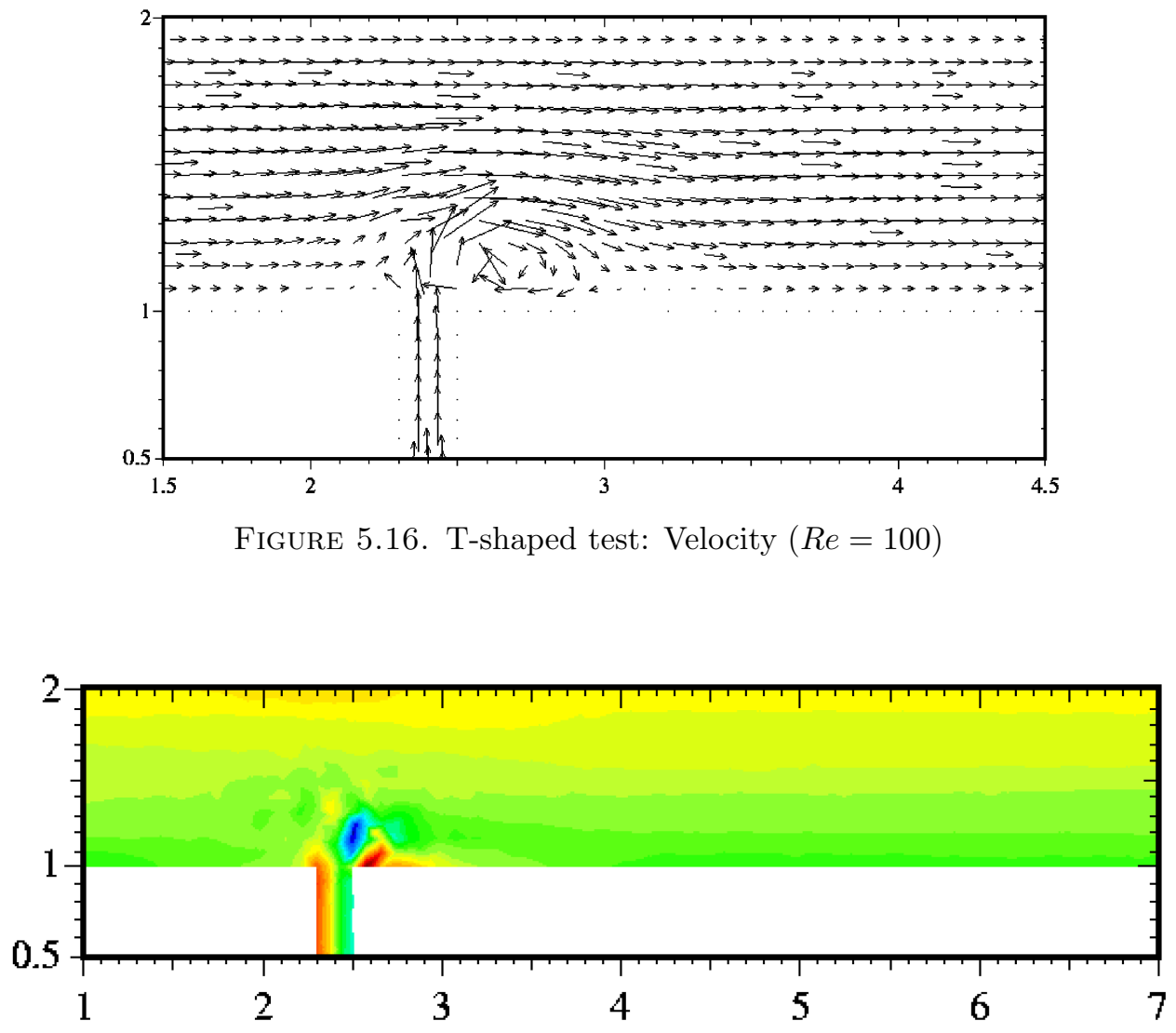

FiguRE 5.17. T-shaped test: Vorticity $(R e=100)$ 


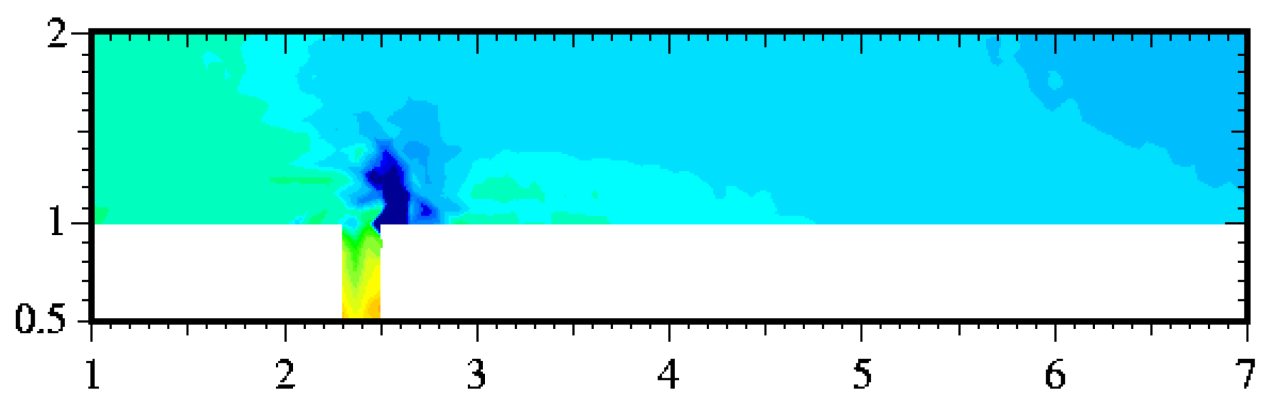

Figure 5.18. T-shaped test: Pressure $(R e=100)$

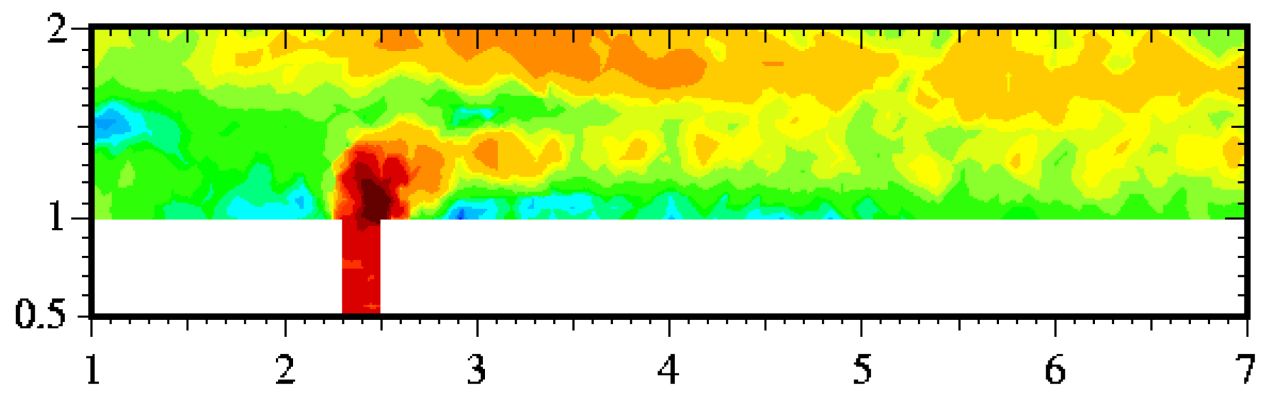

FIgURE 5.19. T-shaped test: Estimator on the initial grid $(R e=10000)$

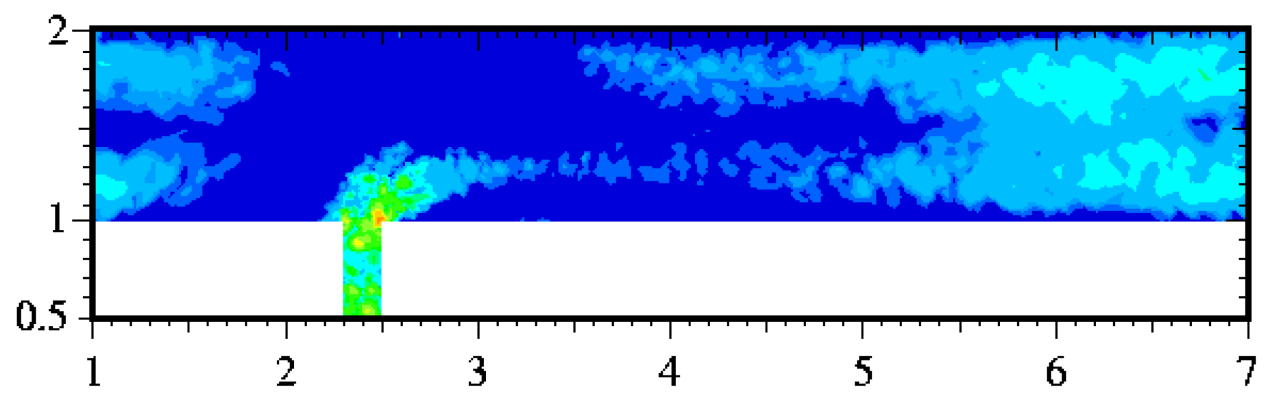

FigurE 5.20. T-shaped test: Estimator on the refined grid $(R e=10000)$

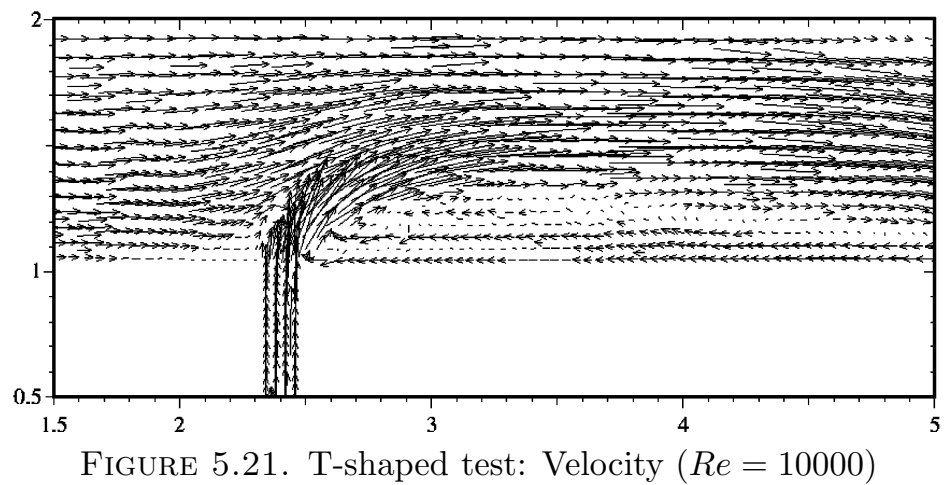




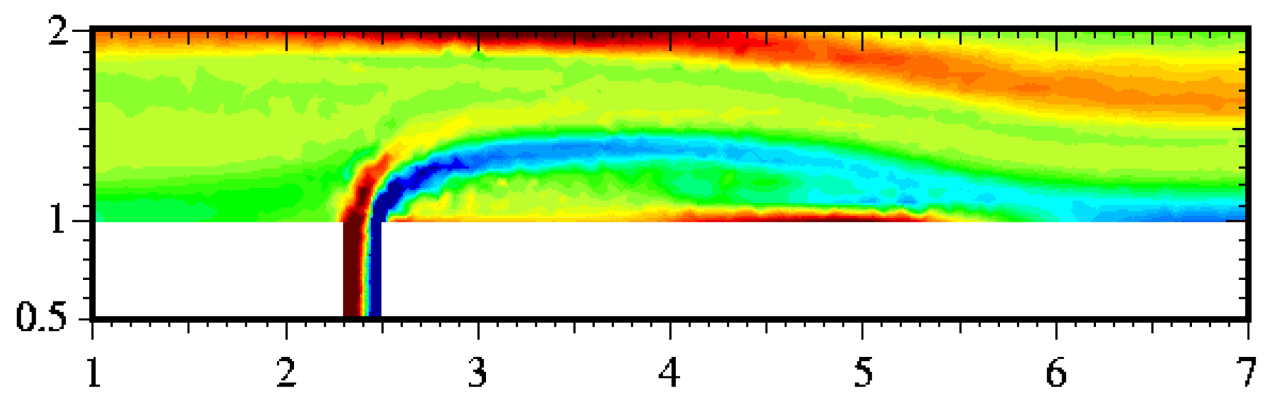

Figure 5.22. T-shaped test: Vorticity $(R e=10000)$

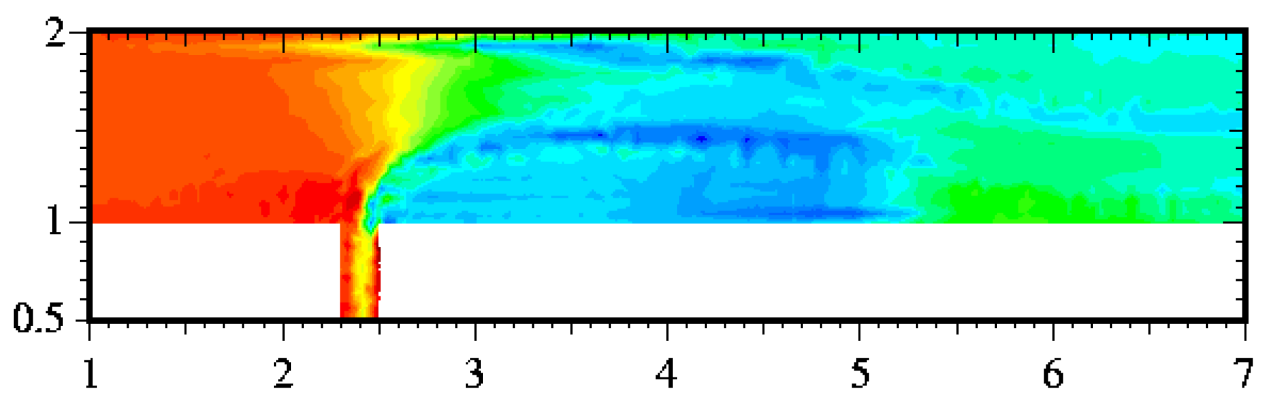

Figure 5.23. T-shaped test: Pressure $(R e=10000)$

\section{ACKNOWLEDGMENT}

The authors would like to thank the referees for their constructive remarks.

\section{REFERENCES}

[1] Amara, M. and Bernardi, C., Convergence of a finite element discretization of the NavierStokes equations in vorticity and stream function formulation, Mathematical Modelling and Numerical Analysis, vol. 33, n. 5, p. 1033-1056 (1999) MR.1726723(2000k:65200)

[2] Amara, M., Capatina-Papaghiuc, D., Trujillo, D.: Stabilized method for the Navier-Stokes equations with nonstandard boundary conditions, Preprint LMA UPPA, n. 0325, p. 1- 29 (2003) (http://lma.univ-pau.fr/publis/publis_pre.php)

[3] Amara, M., Capatina-Papaghiuc, D., Trujillo, D.: A 3D numerical model for the vorticityvelocity-pressure formulation of the Navier-Stokes problem, Preprint LMA UPPA, n. 0510, p.1-11 (2005) (http://lma.univ-pau.fr/publis/publis_pre.php)

[4] Amara, M., Chacon Vera, E. and Trujillo, D.: Vorticity-velocity-pressure formulation for Stokes problem, Math. of Comp., vol. 73, n. 248, p. 1673-1697 (2004) MR2059731 (2005d:65201)

[5] Bernardi, C. and Girault, V.: A local regularization operator for triangular and quadrilateral finite elements, SIAM J. Numer. Anal., vol. 35, n. 5, p. 1893-1916 (1998) MR.1639966 (99g:65107)

[6] Brezzi, F., Fortin, M.: Mixed and Hybrid Finite Element Methods, Springer-Verlag, New York (1991) MR1115205 (92d:65187)

[7] Brezzi, F., Rappaz, J., Raviart, P.-A.: Finite Dimensional Approximation of Nonlinear Problems. Branches of Nonsingular Solutions, Numer. Math., vol. 36, 1-25 (1980) MR595803 (83f:65089a)

[8] Caloz, G., Rappaz, J.: Numerical Analysis for Nonlinear and Bifurcation Problems, Handbook of Numerical Analysis, vol. V, P.G. Ciarlet and J.L. Lions eds, North-Holland, Amsterdam (1997) MR1470227 
[9] Ciarlet, P.: The Finite Element Method for Elliptic Problems, North-Holland (1978) MR0520174 (58:25001)

[10] Conca, C., Pares, C., Pironneau, O. and Thiriet, M.: Navier-Stokes Equations with imposed pressure and velocity fluxes, International Journal for Numerical Methods in Fluids, vol. 20, 267-287 (1995) MR:1316046 (96d:76019)

[11] Costabel, M.: A remark on the regularity of solutions of Maxwell's equations on Lipschitz domains, Mathematical Methods in the Applied Sciences, vol. 12, 365-368 (1990) MR1048563 (91c:35028)

[12] Dubois, F., Salaün, M., Salmon, S.: Vorticity-velocity-pressure and stream function-vorticity formulations for the Stokes problem, J. Math. Pures Appl., vol. 82, p. 1395-1451 (2003) MR2020806 (2004i:35247)

[13] Girault, V. and Raviart, P.-A.: Finite Element Methods for Navier-Stokes Equations. Theory and Algorithms, Springer-Verlag (1986) MR851383 (88b:65129)

[14] Grisvard, P.: Elliptic Problems in Nonsmooth Domains, Pitman (1985) MR775683 (86m:35044)

[15] Pousin, J., Rappaz, J.: Consistency, stability, a priori and a posteriori errors for Petrov-Galerkin methods applied to nonlinear problems, Numer. Math. 69, 213-231 (1994) MR:1310318 (95k:65111)

Laboratoire de Mathématiques Appliquées-CNRS UMR5142, Université de Pau et des PAYS DE L'AdOuR, BP 1155, 64013 PAU CEDEX

E-mail address: mohamed.amara@univ-pau.fr

Laboratoire de Mathématiques Appliquées-CNRS UMR5142, Université de Pau et des PAYs DE L'Adour, BP 1155, 64013 PAU CEDEX

E-mail address: daniela.capatina@univ-pau.dr

Laboratoire de Mathématiques Appliquées-CNRS UMR5142, Université de Pau et des PAYS DE L'Adour, BP 1155, 64013 PAU CEDEX

E-mail address: david.trujillo@univ-pau.fr 